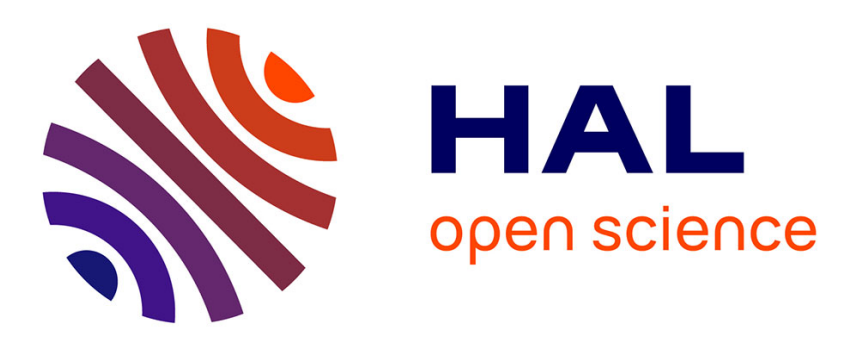

\title{
Laminar shallow viscoplastic fluid flowing through an array of vertical obstacles
}

\author{
Noé Bernabeu, Pierre Saramito, Andrew Harris
}

\section{To cite this version:}

Noé Bernabeu, Pierre Saramito, Andrew Harris. Laminar shallow viscoplastic fluid flowing through an array of vertical obstacles. Journal of Non-Newtonian Fluid Mechanics, 2018, 257, pp.59-70. 10.1016/j.jnnfm.2018.04.001 . hal-01646766v2

\section{HAL Id: hal-01646766 \\ https://hal.science/hal-01646766v2}

Submitted on 19 Mar 2018

HAL is a multi-disciplinary open access archive for the deposit and dissemination of scientific research documents, whether they are published or not. The documents may come from teaching and research institutions in France or abroad, or from public or private research centers.
L'archive ouverte pluridisciplinaire HAL, est destinée au dépôt et à la diffusion de documents scientifiques de niveau recherche, publiés ou non, émanant des établissements d'enseignement et de recherche français ou étrangers, des laboratoires publics ou privés. 


\title{
Laminar shallow viscoplastic fluid flowing through an array of vertical obstacles
}

\author{
Noé Bernabeu, Pierre Saramito, Andrew Harris
}

March 19, 2018

\begin{abstract}
-
A new Bingham-Darcy shallow depth approximation flow model is proposed in this paper. This model is suitable for a shallow viscoplastic fluid flowing on a general topography and crossing an array of vertical obstacles. An analogous porous medium is first introduced to reduce the array of obstacles. The reduction model is based on a continuum model similar to the Brinkman equations, where the usual Darcy model is extended for viscoplastic Bingham fluids. A specific asymptotic analysis of this Bingham-Darcy porous medium for the case of shallow depth flows allows us to produce a new reduced model. Some assumptions are needed for the reduction: laminar flow, small degrees of slope variation of the underlying topography and a yield stress that is small when compared with gravity effects. The resulting solution is a highly nonlinear parabolic equation in terms of the flow height only, and is efficiently solved by a Newton method, without any regularization. However, our numerical predictions compares well, both qualitatively and quantitatively with both experimental measurements and full tridimensional simulations. Finally, a new experiment for a viscoplastic flow over an inclined plane through a network of obstacles is proposed and numerical simulations are provided for future comparison with experiments.
\end{abstract}

\section{Introduction}

The problem of complex fluids flowing through networks of discrete obstacles applies to many applications in natural and material sciences. During natural risk assessments, for example, volcanic debris and/or lava flows may move through dense forests, as was the case for lavas advancing during Kilauea's July 1974 eruption [25] and Etna's 2002-03 eruption [2], among others. To date, lava flow emplacement models have tended to consider tree-free surfaces in completing their simulations (e.g., $[6,19,21])$. The same applies to nonvolcanic debris flows in forested mountainous or urban areas (see e.g. $[15,32]$ ). In terms of material sciences, flow of a viscoplastic fluid through arrays of solid cylinders needs to be considered in industrial processes, such as in the case of fresh concrete spreading through networks of steel bars (see e.g. $[42,43]$ ). 
Taking into account each obstacle in numerical simulations leads to very time consuming computations. The usual approach is to replace the discrete configuration of obstacles with an equivalent continuous medium, the so-called fibrous porous medium. In the case of a Newtonian fluid, this continuous medium is described by the classic Darcy model [14]. This model proposes a linear relation between the flow rate and the pressure drop occurring across the porous medium. In 1949, Brinkman [12] proposed a modification of the classic Darcy model by combining the Navier-Stokes equations with the Darcy model. This combination is useful for situations where there are both flow sub-regions with and without porous media. While the Brinkman model provides a global description of both these sub-regions, the Darcy model alone is unable to describe regions without porous media. Conversely, in the case of a non-Newtonian fluid, the situation is rather complex. This is due to, from one hand, the complexity of the fluid behavior and, on the other hand, the porous micro-structure. Bourgeat and Mikelić [10] proposed a first theoretical analysis of quasi-Newtonian shear-thinning and shear-thickening fluids, and derived a modified-Darcy model. This modified-Darcy model involves an effective viscosity ( $\left.\eta_{\text {eff }}\right)$, which depends both upon the flow rate and the microstructural characteristics of the porous medium, these being described by the permeability tensor $(\boldsymbol{\kappa})$ and porosity $(\phi)$. The porosity is defined as the volume fraction of fluid in the mixture represented by fluid as opposed to the obstacles: it is equal to one for a medium without obstacles and tends to zero as the obstacle density increases. Permeability depends both upon $\phi$ and the geometric configuration of the obstacles, where some explicit expressions of $\boldsymbol{\kappa}$ vs $\phi$ exist, and these depend on a geometrical hypothesis based upon the obstacle distribution. For Non-Newtonian viscoplastic fluids, a yield stress has to be reached to achieve a transition between the unyielded, arrested state of the fluid and the yielding state (see e.g. [36,37]). Thus, there fluids which do not obey to the usual linear Darcy model with their rheological response to an applied stress, as there is also some no-flow situations in porosities [20,34]. Based on an experimental investigation, Pascal [29] proposed - for an Herschel-Bulkley viscoplastic fluid - a modified-Darcy model that uses a threshold gradient. Some papers have since focused on flow through packed beds of spherical particles. Al-Fariss and Pinder [1], for example extended the Pascal [29] model by deriving an equation for the threshold gradient to describe the flow of waxy oil through beds of packed spheres. Chevalier et al. [13], based on experimental measurements for a fluid with a yield stress passing through packed glass beads, proposed an empirical relationship between the pressure drop and the flow rate. Recent studies have considered complex fluid flow through fibrous porous media. Bleyer and Coussot [8], for example performed two-dimensional numerical simulations of a viscoplastic flow through an ordered array of disks; Shahsavari and McKinley [38] investigated the flow of Herschel-Bulkley fluids through fibrous media by means of numerical studies and scaling analyse. As a result, Shahsavari and McKinley [38] developed an effective viscosity function which can be used in the modified-Darcy model for steady fully-developed viscoplastic flows. In addition, Vasilic et al. [42] defined the apparent shear rate using a shift factor and a generalized Brinkman equation. They also performed numerical simulations using a bi-viscosity regularized viscoplastic model and compared their results with experimental measurements on a Carbopol gel flow which was slowly poured into a transparent container where an array of cylindrical steel were located across the middle zone of simulation. Comparing simulations and experimental observations, they obtained both qualitative and quantitative agreements for the final shape of the flow. 
For thin flows, the usual approach is to consider shallow-depth approximations. The shallow-flow approximations of laminar viscoplastic Bingham fluids were first studied by Liu and Mei [26], based on a rigorous asymptotic analysis. This approach was revisited by Balmforth and Craster [4] and extended to the axisymmetric case [3], for an application to volcanic lava domes. For fast flows, such as debris and mud flows on mountain slopes, Laigle and Coussot [24] derived the first reduced model that combines both inertial and viscoplastic effects, where viscoplastic effects are estimated from the friction at the flow base. Assuming a compressible material, Bresch et al. [11] derived a reduced viscoplastic model that also included inertial effects. This approach was next revisited in the incompressible case in terms of asymptotic analysis by Fernández-Nieto et al. [16] and by Ionescu [22] who both applied an augmented Lagrangian algorithm. Practical predictions of natural hazard need to take into account general tridimensional and complex topographies (see e.g. [5]). A new approach for topography in shallow flow models was proposed by Bouchut et al. [9] which relaxes most restrictions, such as slowly varying topographies. Next, Ionescu [23], considered Bingham and Drucker-Prager models and extended this approach with an elegant formulation based on surface differential operators (surface gradient and divergence) while also including inertia effects. For a more exhaustive review of various shallow flow approximations of viscoplastic fluids, see the recent review paper of Saramito and Wachs [37].

The model proposed here allows a shallow-flow approximation of both the BinghamBrinkman model, and involved application a modified-Darcy model for viscoplastic fluids. This model is of practical application for risk assessment, by opening the possibility of numerically investigating the effects of forests on the spatial and temporal of volcanic flow propagation, which has an unknown effect on flow advance (see [18]). Our model could be also useful for industrial processes, such as fresh concrete spreading through arrays of steel bars, as the required computing time is dramatically decreased over fully three-dimensional models. Instead of time-dependent tridimensional simulations with moving free-surfaces, the model presented here requires only the solution of a simple two-dimensional parabolic equation for flow height. The array of obstacles is first reduced to a continuum model by a generalized tensor Brinkman equations for yield strength fluids. Then, by assuming a shallow laminar flow, a low slope variation in the topography and a yield stress that is assumed to be small when compared to the gravity effects, we extend a previous asymptotic analysis [5] to the Brinkman equations extended for the Bingham model.

The outline of this paper is, thus, as follows. Section 2 proposes a new shallow-depth approximation of the viscoplastic Bingham model for a fluid flowing on a general topography and crossing an array of vertical obstacles. Section 3 proposes a Newton's algorithm for efficient solution of an unregularized nonlinear Bingham-Brinkman (reduced) model. Comparisons between numerical simulations and experimental observations are presented and discussed in section 4. Finally, the flow of viscoplastic fluids on an inclined plane through different fibrous mediums is numerically investigated, our aim being to understand and quantify the influence of the obstacles on the flow propagation. This numerical experiment could be reproduced with real fluids for future comparisons and benchmarking. The impatient reader, who is not interested in the asymptotic analysis, can read paragraph 2.1 for the initial problem, then paragraph 2.5 for the final reduced problem and, finally, jump straight to section 4 for a review of our results and a discussion. 


\section{Bingham-Darcy shallow depth approximation}

\subsection{The initial tridimensional problem}

We consider the Bingham model [7] constitutive equation which expresses the deviatoric part $\boldsymbol{\tau}$ of the stress tensor versus the rate of deformation tensor $\dot{\gamma}$ as:

$$
\begin{cases}\boldsymbol{\tau}=\eta \dot{\gamma}+\tau_{y} \frac{\dot{\gamma}}{|\dot{\gamma}|} & \text { when } \dot{\gamma} \neq 0 \\ |\boldsymbol{\tau}| \leqslant \tau_{y} & \text { otherwise. }\end{cases}
$$

where $\eta>0$ is the plastic viscosity and $\tau_{y} \geqslant 0$ is the yield stress. Here $|\boldsymbol{\tau}|=$ $\left((1 / 2) \sum_{i, j=1}^{3} \tau_{i j}^{2}\right)^{1 / 2}$ denotes the conventional norm of a symmetric tensor in mechanics. The total Cauchy stress tensor is $\boldsymbol{\sigma}=-p \cdot \mathbf{I}+\boldsymbol{\tau}$ where $p$ is the pressure and $\mathbf{I}$ the identity tensor. We assume that the array of obstacles can be treated as an equivalent continuum porous medium. The constitutive equation (1) is then completed by the conservations of momentum and mass:

$$
\begin{aligned}
\rho\left(\partial_{t} \mathbf{u}+(\mathbf{u} . \boldsymbol{\nabla}) \mathbf{u}\right)-\operatorname{div}(\boldsymbol{\tau})+\nabla p & =\mathbf{f}_{p}+\rho \mathbf{g}, \\
\operatorname{div} \mathbf{u} & =0
\end{aligned}
$$

where $\rho>0$ is the constant density, $\mathbf{g}$ is the gravity vector and $\mathbf{f}_{p}$ a source term based on local generalized Darcy's law (e.g. see [30]) relating the force exerted on the pore fluid (typically gradient pressure and gravity force) to the macroscopic-scale velocity by:

$$
\mathbf{f}_{p}=-\eta_{\mathrm{eff}} \boldsymbol{\kappa}^{-1} \mathbf{u}
$$

where $\eta_{\text {eff }} \geqslant 0$ is the material local apparent viscosity and $\boldsymbol{\kappa}$ the permeability tensor. The conservation equations with the addition of a Darcy source term in the momentum equation is called the Brinkman equations [12]. This model allows us to deal with mixed cases where only a part of the calculation domain is taken up by a fibrous porous medium. In this case, out side of the porous zone, the permeability is infinite and the source term $\mathbf{f}_{p}$ vanishes to give the standard conservation equations.

It was proved in $[28,31]$ that the permeability tensor is symmetric and positive definite. This means that the permeability tensor has three principal orthogonal axes and three positive principal values. For an arbitrary porous medium, it is possible to find a coordinate system $(x, y, z)$ in which the permeability tensor has a diagonal form. When the medium is anisotropic, at least two elements of the diagonal permeability tensor are not equal. Otherwise, when the does not depend on direction, permeability is isotropic, and the elements of the diagonal permeability tensor are equal. If the distribution of pores or principal directions varies from one point in the medium to another, then the permeability tensor is spatially heterogeneous; otherwise, it is homogeneous.

In our case, with an array of vertical obstacles, the equivalent continuum medium is a fibrous porous one. For convenience, we assume that the $z$-axis is parallel to the fiber axis and that the arrangement of fibers is uniform in each direction perpendicular to the $z$-axis, so $\kappa=\operatorname{diag}\left(\kappa_{\|}, \kappa_{\|}, \kappa_{\perp}\right)$, where $\kappa_{\|}$is the permeability in the direction perpendicular to the fibers and $\kappa_{\perp}$ the permeability in the direction parallel to the fibers. The local strain rate 
is a complex function of the geometry and the fiber arrangement. In the equivalent porous media, it is necessary to define a local effective shear rate $\dot{\gamma}_{\text {eff }}$ which is for the fibrous media, a diagonal tensor where its components are commonly expressed as [39]:

$$
\dot{\gamma}_{\mathrm{eff}, i i}=\frac{\alpha_{i i} \mathbf{u}_{i}}{\sqrt{\kappa_{i i} \phi}}, \forall i \in\{x, y, z\},
$$

where $\boldsymbol{\alpha}=\operatorname{diag}\left(\alpha_{i i}\right)_{i \in\{x, y, z\}}$ denotes the medium dependent shift factor (also called the shape factor) and $\alpha_{i i}$ is the factor in the principal direction $i$. Different expressions are proposed in $[38,43]$ for viscoplastic fluids. We assume that $\boldsymbol{\alpha}$ has the same form as the permeability tensor: $\boldsymbol{\alpha}=\operatorname{diag}\left(\alpha_{\|}, \alpha_{\|}, \alpha_{\perp}\right)$. Let us introduce the tensor $\boldsymbol{A}=$ $\operatorname{diag}\left(\alpha_{\|} / \sqrt{\kappa_{\|} \phi}, \alpha_{\|} / \sqrt{\kappa_{\|} \phi}, \alpha_{\perp} / \sqrt{\kappa_{\perp} \phi}\right)$. When $\dot{\gamma}_{\text {eff }} \neq 0$, which is equivalent to $\mathbf{u} \neq \mathbf{0}$, from the Bingham constitutive equation (1), we obtain the following expression of the effective viscosity:

$$
\begin{aligned}
\eta_{\text {eff }}\left(\dot{\gamma}_{\text {eff }}\right) & =\eta+\frac{\tau_{y}}{\left|\dot{\gamma}_{\text {eff }}\right|} \\
& =\eta+\frac{\tau_{y}}{|\boldsymbol{A u}|},
\end{aligned}
$$

which when combined with the generalized Darcy's law leads, when $\mathbf{u} \neq \mathbf{0}$, to the following expression

$$
\mathbf{f}_{p}=-\left(\frac{\tau_{y}}{|\boldsymbol{A u}|}+\eta\right) \boldsymbol{\kappa}^{-1} \mathbf{u}
$$

In the general case, we obtain a tensor generalization of Pascal's law [29] for an anisotropic fibrous media:

$$
\begin{cases}\mathbf{f}_{p}=-\left(\frac{\tau_{y}}{|\boldsymbol{A u}|}+\eta\right) \boldsymbol{\kappa}^{-1} \mathbf{u}, & \text { when } \mathbf{u} \neq \mathbf{0}, \\ \left|\boldsymbol{A} \boldsymbol{\kappa} \mathbf{f}_{p}\right| \leqslant \tau_{y} & \text { when } \mathbf{u}=\mathbf{0} .\end{cases}
$$

The yield stress $\tau_{y}$ leads to a threshold condition for the transition between "solid" and "fluid" rheological behaviour inside the porous zone in terms of the anisotropic norm $|\boldsymbol{A} \boldsymbol{\kappa} \cdot|$. There are five equations (1)-(5) and four unknowns $\boldsymbol{\tau}, \mathbf{u}, p$ and $\mathbf{f}_{p}$.

We consider a flow over a variable topography passing through a porous zone (see Fig. 1). For any time $t>0$, the flow domain is represented by:

$$
\Lambda(t)=\{(x, y, z) \in \Omega \times \mathbb{R} ; f(x, y)<z<f(x, y)+h(t, x, y)\}
$$

where $\Omega$ is an open and bounded subset of $\mathbb{R}^{2}$. The function $f$ denotes the topography and $h$ the flow vertical height.

Some authors prefer to work with the thickness in a direction normal to the topography and using local curvilinear coordinates (see $[9,23]$ ). It can be easier to carry out an asymptotic analysis with this choice when the effects of the curvature are important or when the variation of the slope is great. In our situation, we will assume that the flow is laminar and that the variation of the slope of the topography is low, so that the Cartesian coordinate system $(x, y, z)$ with a vertical definition of the flow height is a good choice. Furthermore, we focus on the passage of fluid through a network of vertical obstacles modeled by a constant value for the horizontal permeability $\kappa_{\|}$and the vertical permeability $\kappa_{\perp}$, so it 


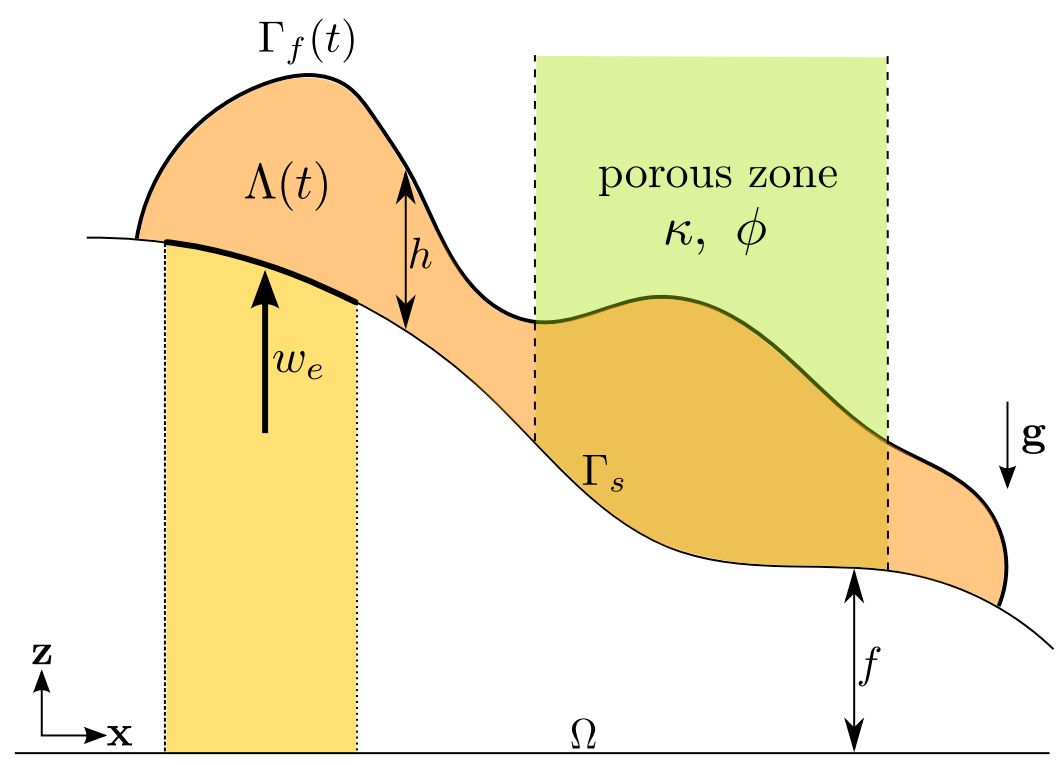

Figure 1: 2D schematic view of a flow on a variable topography, supplied through a vent at the speed $w_{e}$, passing through a porous zone with a permeability $\boldsymbol{\kappa}$ and a porosity $\phi$.

will be easier to write equations in the Cartesian coordinate system $(x, y, z)$ rather than a rotating coordinate system.

The boundary $\partial \Lambda(t)$ of the flow volume $\Lambda(t)$ can be split into three parts (see Fig. 1): the basal topography $\Gamma_{s}$, the flow (upper) free surface $\Gamma_{f}(t)$, and the lateral part $\Gamma_{w}(t)$, as defined by:

$$
\begin{aligned}
\Gamma_{s} & =\{(x, y, z) \in \Omega \times \mathbb{R} ; z=f(x, y)\} \\
\Gamma_{f}(t) & =\{(x, y, z) \in \Omega \times \mathbb{R} ; z=f(x, y)+h(t, x, y)\} \\
\Gamma_{w}(t) & =\{(x, y, z) \in \partial \Omega \times \mathbb{R} ; f(x, y)<z<f(x, y)+h(t, x, y)\}
\end{aligned}
$$

For any $t>0$, the boundary conditions at the flow base are a no-slip condition, an imposed vertical speed and natural zero stress on the free surface:

$$
\begin{aligned}
u_{x}=u_{y}=0 \text { and } u_{z}=w_{e} \text { on } \Gamma_{s} \text { and } \mathbf{u} & =\mathbf{0} \text { on } \Gamma_{w} \\
\boldsymbol{\sigma} \cdot \boldsymbol{\nu} & =0 \text { on } \Gamma_{f}(t)
\end{aligned}
$$

where $\boldsymbol{\nu}$ denotes the unit outward vector of $\partial \Lambda(t)$ on $\Gamma_{f}(t)$. The imposed vertical speed $w_{e}$ corresponds to a supply speed of an injection which in our case is the speed of lava eruption from the vent. Its value is zero beyond the vent.

It remains to describe the evolution of the free surface. It is convenient to introduce the level set function $\varphi$ that expresses as:

$$
\varphi(t, x, y, z)=z-f(x, y)-h(t, x, y) .
$$

Notice that the zero level set, where $\varphi(t, x, y, z)=0$, is exactly at the free surface. The level set function is transported by the flow: $\partial_{t} \varphi+\mathbf{u} \cdot \nabla \varphi=0$. On $\Gamma_{f}(t)$, where $z=f+h$, 
this allows us to write:

$$
\partial_{t} h+u_{x} \partial_{x}(f+h)+u_{y} \partial_{y}(f+h)-u_{z}=0, \forall t>0 \text { and }(x, y) \in \Omega .
$$

This is a first-order transport equation for height $h$ that should be supplemented by an initial condition:

$$
h(t=0, x, y)=h_{\text {init }}(x, y), \forall(x, y) \in \Omega .
$$

where $h_{\text {init }}$ is given. The set of equations is finally completed by an initial condition for the velocity $\mathbf{u}$ :

$$
\mathbf{u}(t=0)=\mathbf{u}_{\text {init }} \text { in } \Lambda(0) \text {. }
$$

To summarize, the three-dimensional problem is composed by the equations (1)-(10) and involves the following unknows: the stress tensor $\boldsymbol{\tau}$, the velocity field $\mathbf{u}$, pressure $p$, the Darcy source $\mathbf{f}_{p}$ and height $h$.

\subsection{Asymptotic shallow analysis}

\section{The dimensionless procedure}

We extend here the asymptotic analysis developed in [5] for a case of tridimensional flow on an arbitrary topography to the case of a flow through a fibrous porous zone. Let $L$ be a characteristic length of the bidimensional domain $\Omega$ and $H$ a characteristic height of the flow. We introduce the dimensionless parameter $\varepsilon=H / L$. Let $U=\rho g H^{3} /(\eta L)$ be a characteristic flow velocity in the $(x, y)$ plane and $g=|\mathbf{g}|$ is the gravity constant. Let $W=\varepsilon U$ be a characteristic velocity in the $z$ direction, $T=L / U$ be a characteristic time, and $P=\rho g H$ be a characteristic pressure. The problem can now be reformulated with dimensionless quantities and unknowns, denoted with tildes:

$$
\begin{array}{r}
x=L \tilde{x}, y=L \tilde{y}, z=H \tilde{z}, t=T \tilde{t}, p=P \tilde{p}, h=H \tilde{h}, \\
u_{x}=U \tilde{u}_{x}, u_{y}=U \tilde{u}_{y}, u_{z}=\varepsilon U \tilde{u}_{z}, w_{e}=\varepsilon U \tilde{w}_{e} \\
f_{p, x}=\frac{\eta U}{\kappa_{\|}} \tilde{f}_{p, x}, f_{p, y}=\frac{\eta U}{\kappa_{\|}} \tilde{f}_{p, y}, f_{p, z}=\varepsilon \frac{\eta U}{\kappa_{\perp}} \tilde{f}_{p, z} .
\end{array}
$$

Our scaling procedure is non-isotropic. The scaling is different for the $z$ coordinate and the $z$ vector component of the velocity vector $\mathbf{u}$ over the $x$ and $y$ ones. The dimensionless rate of deformation tensor $\tilde{\dot{\gamma}}$ is also related to its dimensional counterpart $\dot{\gamma}=\nabla \mathbf{u}+\nabla \mathbf{u}^{T}$ by the following non-isotropic relations:

$$
\begin{aligned}
& \dot{\gamma}_{\alpha \beta}=(U / L) \tilde{\dot{\gamma}}_{\alpha \beta}, \quad \alpha, \beta \in\{x, y\} \\
& \dot{\gamma}_{\alpha z}=(U / H) \tilde{\dot{\gamma}}_{\alpha z}, \quad \alpha \in\{x, y\} \\
& \dot{\gamma}_{z z}=(U / L) \tilde{\dot{\gamma}}_{z z} .
\end{aligned}
$$

The scaling procedure for the deviatoric part of stress $\boldsymbol{\tau}$ is similar:

$$
\begin{aligned}
\tau_{\alpha \beta} & =\eta(U / L) \tilde{\tau}_{\alpha \beta}, \quad \alpha, \beta \in\{x, y\} \\
\tau_{\alpha z} & =\eta(U / H) \tilde{\tau}_{\alpha z}, \quad \alpha \in\{x, y\}, \\
\tau_{z z} & =\eta(U / L) \tilde{\tau}_{z z} .
\end{aligned}
$$




\section{The constitutive equation}

The dimensionless rate of deformation tensor can be expressed versus the dimensionless velocity as:

$$
\begin{aligned}
\tilde{\dot{\gamma}}_{\alpha \beta} & =\partial_{\tilde{\beta}} \tilde{u}_{\alpha}+\partial_{\tilde{\alpha}} \tilde{u}_{\beta}, \quad \alpha, \beta \in\{x, y\}, \\
\tilde{\dot{\gamma}}_{\alpha z} & =\partial_{\tilde{z}} \tilde{u}_{\alpha}+\varepsilon^{2} \partial_{\tilde{\alpha}} \tilde{u}_{z}, \quad \alpha \in\{x, y\}, \\
\tilde{\dot{\gamma}}_{z z} & =2 \partial_{\tilde{z}} \tilde{u}_{z} .
\end{aligned}
$$

The tensor norm scales as: $|\dot{\gamma}|=(U / H) E$, where by using (3), we get:

$$
\begin{aligned}
E= & \left\{\varepsilon^{2}\left(\partial_{\tilde{x}} \tilde{u}_{y}+\partial_{\tilde{y}} \tilde{u}_{x}\right)^{2}+2 \varepsilon^{2}\left(\partial_{\tilde{x}} \tilde{u}_{x}\right)^{2}+2 \varepsilon^{2}\left(\partial_{\tilde{y}} \tilde{u}_{y}\right)^{2}\right. \\
& \left.+2 \varepsilon^{2}\left(\partial_{\tilde{x}} \tilde{u}_{x}+\partial_{\tilde{y}} \tilde{u}_{y}\right)^{2}+\left(\partial_{\tilde{z}} \tilde{u}_{x}+\varepsilon^{2} \partial_{\tilde{x}} \tilde{u}_{z}\right)^{2}+\left(\partial_{\tilde{z}} \tilde{u}_{y}+\varepsilon^{2} \partial_{\tilde{y}} \tilde{u}_{z}\right)^{2}\right\}^{\frac{1}{2}}
\end{aligned}
$$

Let us introduce the Bingham dimensionless number $B i$ that compares the yield stress $\tau_{y}$ to a characteristic viscous stress $\eta U / H$ :

$$
B i=\frac{\tau_{y} H}{\eta U}=\varepsilon^{-1} \frac{\tau_{y}}{\rho g H} .
$$

We suppose that $B i=\mathcal{O}(1)$ in $\varepsilon$. This hypothesis means that $\tau_{y} /(\rho g H)=\mathcal{O}(\varepsilon)$ or equivalently that the yield stress $\tau_{y}$ is assumed to be small when compared to the gravity effects $\rho g H$. This assumption can be tested using the ratio of yield strength to basal shear stress using values from natural (lava flow) cases. Using the data of Robert et al. [33] we have, for a lava flow active on Kilauea (Hawaii) in 1974, a typical yield strength of 250 $\mathrm{Pa}$, and a typical flow depth of $5.7 \mathrm{~m}$, giving a basal shear stress (given the underlying slope of 3.5 degrees and typical lava density of $1000 \mathrm{~kg} / \mathrm{m}^{3}$ ) of $3600 \mathrm{~Pa}$. Thus, the ratio of yield strength to basal shear stress is 0.07 , supporting the assumption that yield stress is small when compared to the gravity effects.

When $|\boldsymbol{\tau}| \geqslant \tau_{y}$ we obtain a dimensionless version of the constitutive equation (1):

$$
\tilde{\tau}_{i j}=\left(\frac{B i}{E}+1\right) \tilde{\dot{\gamma}}_{i j}
$$

Then $|\boldsymbol{\tau}|=\eta(U / H) T$ where:

$$
T=\left\{\tilde{\tau}_{x z}^{2}+\tilde{\tau}_{y z}^{2}+\frac{1}{2} \varepsilon^{2} \tilde{\tau}_{x x}^{2}+\frac{1}{2} \varepsilon^{2} \tilde{\tau}_{y y}^{2}+\frac{1}{2} \varepsilon^{2} \tilde{\tau}_{z z}^{2}+\varepsilon^{2} \tilde{\tau}_{x y}^{2}\right\}^{\frac{1}{2}} .
$$

The von Mises condition $|\boldsymbol{\tau}| \leqslant \tau_{y}$ then becomes $T \leqslant B i$. The constitutive equation (1) can now be written:

$$
\begin{cases}\tilde{\tau}=\left[\frac{B i}{E}+1\right] \tilde{\dot{\gamma}} & \text { when } E \neq 0, \\ T \leqslant B i & \text { otherwise. }\end{cases}
$$

\section{The Darcy source term equation}

The anisotropic norm of velocity scales as: $|\boldsymbol{A u}|=|\boldsymbol{A}| U V$ where:

$$
V=\frac{\left(A_{x x}^{2} \tilde{u}_{x}^{2}+A_{y y}^{2} \tilde{u}_{y}^{2}+\varepsilon^{2} A_{z z}^{2} \tilde{u}_{z}^{2}\right)^{1 / 2}}{|\boldsymbol{A}|} .
$$


Let us introduce the Darcy-Bingham dimensionless number $B i_{p}$ that compares the yield stress $\tau_{y}$ to a characteristic viscous stress in fibrous porous medium $\eta U|\boldsymbol{A}|$ :

$$
B i_{p}=\frac{\tau_{y}}{\eta U|\boldsymbol{A}|}=\frac{1}{H|\boldsymbol{A}|} B i .
$$

We suppose that $B i_{p}=\mathcal{O}(1)$ in $\varepsilon$. This hypothesis means that $\tau_{y}|\boldsymbol{A}| /(\rho g)=\mathcal{O}(\varepsilon)$ or equivalently that the yield stress $\tau_{y}$ is supposed to be small when compared to the gravity effects in a porous medium $\rho g /|\boldsymbol{A}|$. When $\mathbf{u} \neq \mathbf{0}$ we obtain a dimensionless version of the Darcy source term equation (5):

$$
\tilde{\mathbf{f}}_{p}=-\left(\frac{B i_{p}}{V}+1\right) \tilde{\mathbf{u}}
$$

The Darcy source term norm scales as: $\left|\boldsymbol{A} \boldsymbol{\kappa} \mathbf{f}_{p}\right|=|\boldsymbol{A}| \eta U F$ where

$$
F=\frac{\left(A_{x x}^{2} \tilde{f}_{p, x}^{2}+A_{y y}^{2} \tilde{f}_{p, y}^{2}+\varepsilon^{2} A_{z z}^{2} \tilde{f}_{p, z}^{2}\right)^{1 / 2}}{|\boldsymbol{A}|} .
$$

Then, the threshold condition $\left|\boldsymbol{A} \boldsymbol{\kappa} \mathbf{f}_{p}\right| \leqslant \tau_{y}$ becomes $F \leqslant B i_{p}$ and the Darcy source term equation (5) can be written as:

$$
\begin{cases}\tilde{\mathbf{f}}_{p}=-\left(\frac{B i_{p}}{V}+1\right) \tilde{\mathbf{u}}, & \text { when } \tilde{\mathbf{u}} \neq \mathbf{0}, \\ F \leqslant B i_{p} & \text { when } \tilde{\mathbf{u}}=\mathbf{0},\end{cases}
$$

\section{The conservation equations}

Let us introduce the Reynolds number and Darcy numbers:

$$
R e=\frac{\rho U L}{\eta}=\frac{\rho^{2} g H^{3}}{\eta^{2}}, \quad D a_{\|}=\frac{\kappa_{\|}}{H^{2}}, \quad D a_{\perp}=\frac{\kappa_{\perp}}{H^{2}} .
$$

We suppose that $R e=\mathcal{O}(1)$ in $\varepsilon$ which means that the flow is assumed to be sufficiently slow for the inertia effects to be neglected at the zeroth order of development in $\varepsilon$. We assume also that $D a_{\|}=\mathcal{O}(1)$ and $D a_{\perp}=\mathcal{O}(1)$ which means that the presence of a porous medium has an influence on the horizontal flow, but has no influence for the vertical flow.

The conservation of momentum and mass (2)-(3) become:

$$
\begin{aligned}
\varepsilon^{2} \operatorname{Re}\left(\partial_{\tilde{t}} \tilde{u}_{x}+\tilde{u}_{x} \partial_{\tilde{x}} \tilde{u}_{x}+\tilde{u}_{y} \partial_{\tilde{y}} \tilde{u}_{x}+\tilde{u}_{z} \partial_{\tilde{z}} \tilde{u}_{x}\right)=- & \partial_{\tilde{x}} \tilde{p}+\varepsilon^{2}\left(\partial_{\tilde{x}} \tilde{\tau}_{x x}+\partial_{\tilde{y}} \tilde{\tau}_{x y}\right) \\
& +\partial_{\tilde{z}} \tilde{\tau}_{x z}+D a_{\|}^{-1} \tilde{f}_{p, x}, \\
\varepsilon^{2} \operatorname{Re}\left(\partial_{\tilde{t}} \tilde{u}_{y}+\tilde{u}_{x} \partial_{\tilde{x}} \tilde{u}_{y}+\tilde{u}_{y} \partial_{\tilde{y}} \tilde{u}_{y}+\tilde{u}_{z} \partial_{\tilde{z}} \tilde{u}_{y}\right)=- & \partial_{\tilde{y}} \tilde{p}+\varepsilon^{2}\left(\partial_{\tilde{x}} \tilde{\tau}_{x y}+\partial_{\tilde{y}} \tilde{\tau}_{y y}\right) \\
& +\partial_{\tilde{z}} \tilde{\tau}_{y z}+D a_{\|}^{-1} \tilde{f}_{p, y}, \\
\varepsilon^{4} \operatorname{Re}\left(\partial_{\tilde{t}} \tilde{u}_{z}+\tilde{u}_{x} \partial_{\tilde{x}} \tilde{u}_{\tilde{z}}+\tilde{u}_{y} \partial_{\tilde{y}} \tilde{u}_{\tilde{z}}+\tilde{u}_{z} \partial_{\tilde{z}} \tilde{u}_{z}\right)=- & \partial_{\tilde{z}} \tilde{p}+\varepsilon^{2}\left(\partial_{\tilde{x}} \tilde{\tau}_{x z}+\partial_{\tilde{y}} \tilde{\tau}_{y z}+\partial_{\tilde{z}} \tilde{\tau}_{z z}\right) \\
& -1+\varepsilon D a_{\perp}^{-1} \tilde{f}_{p, z}, \\
\partial_{\tilde{x}} \tilde{u}_{x}+\partial_{\tilde{y}} \tilde{u}_{y}+\partial_{\tilde{z}} \tilde{u}_{z}=0 . &
\end{aligned}
$$




\section{Boundary and initial conditions}

The no-slip boundary condition (6) can be written:

$$
\tilde{u}_{x}=\tilde{u}_{y}=0 \text { and } \tilde{u}_{z}=\tilde{w}_{e} \text { on } \Gamma_{s} \text { and } \tilde{\mathbf{u}}=\mathbf{0} \text { on } \Gamma_{w}
$$

The unit outward normal $\boldsymbol{\nu}$ on the free surface $\Gamma_{s}(t)$ is expressed as:

$$
\boldsymbol{\nu}=\frac{\boldsymbol{\nabla} \varphi}{\|\boldsymbol{\nabla} \varphi\|}=\frac{1}{\sqrt{1+\mid \boldsymbol{\nabla}(f+h)^{2}}}\left(\begin{array}{c}
-\partial_{x}(f+h) \\
-\partial_{y}(f+h) \\
1
\end{array}\right) .
$$

Then (7) becomes:

$$
\left(\begin{array}{ccc}
\tau_{x x}-p & \tau_{x y} & \tau_{x z} \\
\tau_{x y} & \tau_{y y}-p & \tau_{y z} \\
\tau_{x z} & \tau_{y z} & \tau_{z z}-p
\end{array}\right)\left(\begin{array}{c}
-\partial_{x}(f+h) \\
-\partial_{y}(f+h) \\
1
\end{array}\right)=\left(\begin{array}{l}
0 \\
0 \\
0
\end{array}\right) .
$$

which, in dimensionless form, is:

$$
\begin{aligned}
& -\left(\varepsilon^{2} \tilde{\tau}_{x x}-\tilde{p}\right) \partial_{\tilde{x}}(\tilde{f}+\tilde{h})-\varepsilon^{2} \tilde{\tau}_{x y} \partial_{\tilde{y}}(\tilde{f}+\tilde{h})+\tilde{\tau}_{x z}=0 \\
& -\varepsilon^{2} \tilde{\tau}_{x y} \partial_{\tilde{x}}(\tilde{f}+\tilde{h})-\left(\varepsilon^{2} \tilde{\tau}_{y y}-\tilde{p}\right) \partial_{\tilde{y}}(\tilde{f}+\tilde{h})+\tilde{\tau}_{y z}=0 \\
& -\varepsilon^{2} \tilde{\tau}_{x z} \partial_{\tilde{x}}(\tilde{f}+\tilde{h})-\varepsilon^{2} \tilde{\tau}_{y z} \partial_{\tilde{y}}(\tilde{f}+\tilde{h})+\varepsilon^{2} \tilde{\tau}_{z z}-\tilde{p}=0
\end{aligned}
$$

where $\tilde{f}=f / H$ denotes the dimensionless topography and is known. The transport equation (8) for the flow height $h$ becomes:

$$
\partial_{\tilde{t}} \tilde{h}+\tilde{u}_{x} \partial_{\tilde{x}}(\tilde{f}+\tilde{h})+\tilde{u}_{y} \partial_{\tilde{y}}(\tilde{f}+\tilde{h})-\tilde{u}_{z}=0 .
$$

The dimensionless problem is completed by the initial conditions for the dimensionless height and velocity. The initial (1)-(10) problem and its dimensionless version are equivalent, since the change of unknowns is simply linear.

\subsection{The zeroth order approximation}

In this section, we only consider the dimensionless problem: since there is no ambiguity, we omit the tilde on the dimensionless variables. We assume that the unknowns admit the following development in $\varepsilon$ when $\varepsilon \ll 1$ :

$$
\begin{aligned}
\boldsymbol{\tau} & =\boldsymbol{\tau}_{0}+\varepsilon \boldsymbol{\tau}_{1}+\varepsilon^{2} \boldsymbol{\tau}_{2}+\ldots \\
\mathbf{u} & =\mathbf{u}_{0}+\varepsilon \mathbf{u}_{1}+\varepsilon^{2} \mathbf{u}_{2}+\ldots \\
p & =p_{0}+\varepsilon p_{1}+\varepsilon^{2} p_{2}+\ldots \\
h & =h_{0}+\varepsilon h_{1}+\varepsilon^{2} h_{2}+\ldots \\
\mathbf{f}_{p} & =\mathbf{f}_{p, 0}+\varepsilon \mathbf{f}_{p, 1}+\varepsilon^{2} \mathbf{f}_{p, 2}+\ldots
\end{aligned}
$$

In this section, next aim is to obtain the problem at the zero order for $\boldsymbol{\tau}_{0}, \mathbf{u}, p_{0}$ and $h_{0}$. Since we only consider the zeroth order, we also omit the zero subscript in this paragraph. Let us denote $\boldsymbol{\nabla}_{\|}=\left(\partial_{x}, \partial_{y}\right)$ the gradient vector in the $O x y$ plane, $\mathbf{u}_{\|}=\left(u_{x}, u_{y}\right)$ and 
$\mathbf{f}_{p, \|}=\left(f_{p, x}, f_{p, y}\right)$, the projected velocity and Darcy's force in this plane and $\boldsymbol{\tau}_{\|}=\left(\tau_{x z}, \tau_{y z}\right)$ the shear stress vector in the same plane. For any $\mathbf{v}_{\|}=\left(v_{x}, v_{y}\right)$ we also denote as $\operatorname{div}_{\|} \mathbf{v}_{\|}=\partial_{x} v_{x}+\partial_{y} v_{y}$ the corresponding plane divergence and $\left|\mathbf{v}_{\|}\right|=\left(v_{x}^{2}+v_{y}^{2}\right)^{1 / 2}$ the usual Euclidean norm in $\mathbb{R}^{2}$. For convenience, we also denote as $\operatorname{dir}\left(\mathbf{v}_{\|}\right)=\mathbf{v}_{\|} /\left|\mathbf{v}_{\|}\right|$the direction of any nonzero plane vector. With these notations, we have $E=\left|\partial_{z} \mathbf{u}_{\|}\right|, T=\left|\boldsymbol{\tau}_{\|}\right|$ and $V=\frac{A_{\|}}{|\boldsymbol{A}|}\left|\mathbf{u}_{\|}\right|, F=\frac{A_{\|}}{|\boldsymbol{A}|}\left|\mathbf{f}_{p, \|}\right|$ at the zeroth order, where $A_{\|}:=A_{x x}=A_{y y}=\frac{\alpha_{\|}}{\sqrt{\kappa_{\|} \phi}}$. The constitutive equation (11) then reduces to:

$$
\begin{aligned}
\tau_{\alpha z} & =\left[\frac{B i}{\left|\partial_{z} \mathbf{u}_{\|}\right|}+1\right] \partial_{z} u_{\alpha}, \quad \forall \alpha \in\{x, y\} \\
\tau_{\alpha \beta} & =\left[\frac{B i}{\left|\partial_{z} \mathbf{u}_{\|}\right|}+1\right]\left(\partial_{\beta} u_{\alpha}+\partial_{\alpha} u_{\beta}\right), \quad \forall \alpha, \beta \in\{x, y\} \\
\tau_{z z} & =2\left[\frac{B i}{\left|\partial_{z} \mathbf{u}_{\|}\right|}+1\right] \partial_{z} u_{z},
\end{aligned}
$$

when $\partial_{z} \mathbf{u}_{\|} \neq \mathbf{0}$ and

$$
\left|\boldsymbol{\tau}_{\|}\right| \leqslant B i, \text { otherwise. }
$$

The Darcy's force equation (12) then reduces to:

$$
\begin{cases}\mathbf{f}_{p}=-\left(\frac{B i_{p, \|}}{\left|\mathbf{u}_{\|}\right|}+1\right) \mathbf{u}, & \text { when } \mathbf{u}_{\|} \neq \mathbf{0} \\ \left|\mathbf{f}_{p, \|}\right| \leqslant B i_{p, \|} & \text { when } \mathbf{u}_{\|}=\mathbf{0}\end{cases}
$$

where $B i_{p, \|}=\frac{|\boldsymbol{A}|}{A_{\|}} B i_{p}=\frac{\sqrt{\kappa_{\|} \phi}}{H \alpha_{\|}} B i$.

From the conservation laws (13) we obtain at the zeroth order:

$$
\begin{aligned}
\partial_{z} \tau_{x z}-\partial_{x} p+D a_{\|}^{-1} f_{p, x} & =0, \\
\partial_{z} \tau_{y z}-\partial_{y} p+D a_{\|}^{-1} f_{p, y} & =0, \\
\partial_{z} p & =-1, \\
\partial_{x} u_{x}+\partial_{y} u_{y}+\partial_{z} u_{z} & =0 .
\end{aligned}
$$

The free surface boundary condition (14) at $z=f(x, y)+h(t, x, y)$ reduces at the zeroth order to:

$$
\begin{aligned}
\tau_{x z}+p \partial_{x}(f+h) & =0 \\
\tau_{y z}+p \partial_{y}(f+h) & =0, \\
p & =0 .
\end{aligned}
$$

The velocity condition at the base $(z=f(x, y))$ remains unchanged but using the projected velocity, its can be written: 


$$
\begin{aligned}
& \mathbf{u}_{\|}=\mathbf{0}, \\
& u_{z}=w_{e} .
\end{aligned}
$$

The others equations, i.e. the transport equation (15), the no-slip boundary condition on walls and the initial conditions for $\mathbf{u}$ and $h$ remains unchanged at the zeroth order.

\subsection{Reducing the problem}

We can now show that the zeroth order problem reduces to a nonlinear parabolic problem involving only one unknown $h$. All other quantities $\boldsymbol{\tau}, \mathbf{u}, p$ and $\mathbf{f}_{p}$ at the zeroth order can be explicitly computed from $h$.

From (19) we get at the free surface $z=f+h$ :

$$
\begin{aligned}
p(z=f+h) & =0, \\
\boldsymbol{\tau}_{\|}(z=f+h) & =\mathbf{0} .
\end{aligned}
$$

Integrating (18c) in $z^{\prime}$ from $z^{\prime}=z$ to $z^{\prime}=f+h$ and using (21a), we have:

$$
p(t, x, y, z)=f(x, y)+h(t, x, y)-z .
$$

As $f$ is known, the quantity $p$ depends only upon the unknown $h$. From the conservation laws (18a)-(18b) and the pressure expression (22), we obtain:

$$
\partial_{z} \boldsymbol{\tau}_{\|}+D a_{\|}^{-1} \mathbf{f}_{p, \|}=\nabla_{\|}(f+h) .
$$

To find more easily an expression of $\mathbf{u}_{\|}$involving the only unknown $h$ from the set of equations, we can make some assumptions on the velocity profile $\mathbf{u}_{\|}$and shear stress vector $\boldsymbol{\tau}$ based on the profiles obtained in [5] given by equations (21) and (18). We assume that the solutions have the same form: $\mathbf{u}_{\|}=-g(z) \boldsymbol{\nabla}_{\|}(f+h)$ where the function $g$ is in $\mathcal{C}^{1}\left([f, f+h], \mathbb{R}^{+}\right) \cap \mathcal{D}^{2}\left([f, f+h], \mathbb{R}^{+}\right)$, and $\boldsymbol{\tau}_{\|}=-\tau(z) \boldsymbol{\nabla}_{\|}(f+h)$ where the function $\tau$ is in $\mathcal{C}^{1}\left([f, f+h], \mathbb{R}^{+}\right)$. Let us first deal with the particular case where $\boldsymbol{\nabla}_{\|}(f+h)=\mathbf{0}$. In this case, $\mathbf{u}_{\|}=\mathbf{0}, \boldsymbol{\tau}_{\|}=\mathbf{0}$ and by $(23), \mathbf{f}_{p, \|}=\mathbf{0}$. Otherwise, when $\boldsymbol{\nabla}_{\|}(f+h) \neq \mathbf{0}$, we assume that there exits a critical height $h_{c}$ in $[0, h]$ for which the function $g$ strictly increases on $\left[f, f+h_{c}\right]$, is constant on $\left.] f+h_{c}, f+h\right]$ and for which the function $\tau$ strictly decreases on $\left[f, f+h_{c}\right]$ and satisfies:

$$
\tau\left(f+h_{c}\right)=\frac{B i}{\left|\nabla_{\|}(f+h)\right|} .
$$

For all points $(x, y) \in \Omega$, as $g$ is strictly increasing on $[f, f+h]$, constant on $\left.] f+h_{c}, f+h\right]$ and $\mathbf{u}_{\|}(z=f)=\mathbf{0}$, thus an equivalent condition of $\mathbf{u}_{\|}=\mathbf{0}, \forall z \in[f, f+h]$ is $h_{c}=0$. From equation (16a), the von Mises condition (16d) and assumptions on $g$, we obtain:

$$
\begin{cases}\boldsymbol{\tau}_{\|}=-\left(\frac{B i}{\left|\nabla_{\|}(f+h)\right|}+g^{\prime}(z)\right) \nabla_{\|}(f+h), & \text { when } z \in\left[f, f+h_{c}\right], \\ \left|\boldsymbol{\tau}_{\|}\right| \leqslant B i, & \text { when } \left.z \in] f+h_{c}, f+h\right] .\end{cases}
$$


From the Darcy's force equation (17) projected on the plane $O x y$ and assumptions on $g$, we obtain:

$$
\begin{cases}\mathbf{f}_{p, \|}(z)=\left(\frac{B i_{p, \|}}{\left|\boldsymbol{\nabla}_{\|}(f+h)\right|}+g(z)\right) \nabla_{\|}(f+h), & \text { when } h_{c}>0 \\ \left|\mathbf{f}_{p, \|}\right| \leqslant B i_{p, \|} & \text { when } h_{c}=0\end{cases}
$$

Let us describe equation (23) for the case where $h_{c}>0$. By using the expression of $\boldsymbol{\tau}_{\|}$ (25) and the expression of $\mathbf{f}_{p, \|}(26)$, this gives the following scalar equations

$$
\begin{gathered}
g^{\prime \prime}(z)-D a_{\|}^{-1} g(z)=\frac{D a_{\|}^{-1} B i_{p, \|}}{\left|\nabla_{\|}(f+h)\right|}-1, \text { when } z \in\left[f, f+h_{c}\right], \\
\left.\left.\tau^{\prime}(z)=D a_{\|}^{-1}\left(g\left(f+h_{c}\right)+\frac{B i_{p, \|}}{\left|\nabla_{\|}(f+h)\right|}\right)-1, \text { when } z \in\right] f+h_{c}, f+h\right] .
\end{gathered}
$$

Equation (27) is a second-order linear ODE with $z$-independent coefficients, with a discriminant $\Delta=4 D a_{\|}^{-1}$. Thus, we can define $\alpha$ and $\beta$ independent of $z$ such that:

$g(z)=\alpha \exp \left(D a_{\|}^{-1 / 2} z\right)+\beta \exp \left(-D a_{\|}^{-1 / 2} z\right)+D a_{\|}\left(1-\frac{D a_{\|}^{-1} B i_{p, \|}}{\left|\boldsymbol{\nabla}_{\|}(f+h)\right|}\right)$, for $z \in\left[f, f+h_{c}\right]$

Here, $\alpha, \beta$ are determined by the boundary conditions. The no-slip condition on the basal topography write:

$$
g(f)=0
$$

The assumption that $g$ is constant on $\left.] f+h_{c}, f+h\right]$ and $\mathcal{C}^{1}$ on $[f, f+h]$ leads to the second condition when $h_{c}<h$ :

$$
g^{\prime}\left(f+h_{c}\right)=0
$$

When $h_{c}=h$, condition (30) remains valid. Indeed, from the value of $\tau$ in $z=f+h_{c}=f+h$ given by (24) and the boundary condition (21b), we obtain $B i=0$. Thus by using the expression of $\boldsymbol{\tau}_{\|}$in $z=f+h_{c}=f+h$ given in (25), we arrive at equation (30).

Writing these boundary conditions with the expression of $g(29)$ leads to:

$$
\begin{aligned}
\alpha \exp \left(D a_{\|}^{-1 / 2} f\right)+\beta \exp \left(-D a_{\|}^{-1 / 2} f\right) & =D a_{\|}\left(\frac{D a_{\|}^{-1} B i_{p, \|}}{\left|\nabla_{\|}(f+h)\right|}-1\right), \\
\alpha \exp \left(D a_{\|}^{-1 / 2}\left(f+h_{c}\right)\right)-\beta \exp \left(-D a_{\|}^{-1 / 2}\left(f+h_{c}\right)\right) & =0,
\end{aligned}
$$

This linear system in terms of $\alpha$ and $\beta$ now solves as: 


$$
\left\{\begin{array}{l}
\alpha=D a_{\|}\left(\frac{D a_{\|}^{-1} B i_{p, \|}}{\left|\nabla_{\|}(f+h)\right|}-1\right) \frac{\exp \left(-D a_{\|}^{-1 / 2}\left(f+h_{c}\right)\right)}{2 \cosh \left(D a_{\|}^{-1 / 2} h_{c}\right)} \\
\beta=D a_{\|}\left(\frac{D a_{\|}^{-1} B i_{p, \|}}{\left|\nabla_{\|}(f+h)\right|}-1\right) \frac{\exp \left(D a_{\|}^{-1 / 2}\left(f+h_{c}\right)\right)}{2 \cosh \left(D a_{\|}^{-1 / 2} h_{c}\right)}
\end{array}\right.
$$

It remains to characterize from the input data an equivalent condition of $h_{c}<h$ and a relation to determine $h_{c}$. We have already seen that $h_{c}=h$ implies $B i=0$. It can be proved that the reverse is true. Indeed, suppose that $B i=0$. From (24) and (21b), $\boldsymbol{\tau}_{\|}\left(f+h_{c}\right)=\boldsymbol{\tau}_{\|}(f+h)=\mathbf{0}$. By integrate between $f+h_{c}$ and $f+h$ in equation (23) and using the expression of Darcy's force given in (26), we arrive at:

$$
\int_{f+h_{c}}^{f+h} g(z) \mathrm{d} z=D a_{\|}\left(h-h_{c}\right)
$$

The function $g$ is constant on the interval $\left[f+h_{c}, f+h\right]$. Thus, from the expression of $g$ (29), for all $z \in\left[f+h_{c}, f+h\right]$,

$$
g(z)=g\left(f+h_{c}\right)=D a_{\|}-\frac{D a_{\|}}{\cosh \left(D a_{\|}^{-1 / 2} h_{c}\right)}
$$

Finally, from the balance of $(35), h_{c}=h$, proving the equivalence: $h_{c}=h \Leftrightarrow B i=0$. It remains to determine $h_{c}$ from the input data when $B i \neq 0 \Leftrightarrow h_{c} \in[0, h[$. By integrating equation (28) between $f+h_{c}$ and $f+h$ and using (21b), we obtain:

$$
\left(h-h_{c}\right) g\left(f+h_{c}\right)=\left(D a_{\|}-\frac{B i_{p, \|}}{\left|\nabla_{\|}(f+h)\right|}\right)\left(h-h_{c}\right)-\frac{D a_{\|} B i}{\left|\nabla_{\|}(f+h)\right|} .
$$

Writing this equation with the expression of $g$ given in (29) gives:

$$
\left(h-h_{c}\right)\left(\alpha \exp \left(D a_{\|}^{-1 / 2}\left(f+h_{c}\right)\right)+\beta \exp \left(-D a_{\|}^{-1 / 2}\left(f+h_{c}\right)\right)\right)=-\frac{D a_{\|} B i}{\left|\nabla_{\|}(f+h)\right|} .
$$

Finally, using the expressions of $\alpha$ and $\beta$ from (33) and (34) in the previous equation, we obtain a relation on $h_{c}$ :

$$
B i \cosh \left(D a_{\|}^{-1 / 2} h_{c}\right)=\left(h-h_{c}\right)\left(\left|\nabla_{\|}(f+h)\right|-D a_{\|}^{-1} B i_{p, \|}\right)
$$

This expression gives a necessary condition so that $h_{c}>0$. Because $B i \cosh \left(D a_{\|}^{-1 / 2} h_{c}\right)$ and $\left(h-h_{c}\right)$ are strictly positives when $0<h_{c}<h$, necessarily, $\left|\nabla_{\|}(f+h)\right|-D a_{\|}^{-1} B i_{p, \|}>$ 
0. The inequation is still true when $h_{c}=h$ because in this case $B i_{p, \|}=0$. We deduce a more general necessary condition:

$$
\begin{aligned}
h_{c}>0 & \Rightarrow h_{c}\left(\left|\nabla_{\|}(f+h)\right|-D a_{\|}^{-1} B i_{p, \|}\right)>0 \\
& \Rightarrow h\left(\left|\nabla_{\|}(f+h)\right|-D a_{\|}^{-1} B i_{p, \|}\right)>B i \cosh \left(D a_{\|}^{-1 / 2} h_{c}\right) \\
& \Rightarrow h\left(\left|\nabla_{\|}(f+h)\right|-D a_{\|}^{-1} B i_{p, \|}\right)>B i
\end{aligned}
$$

The reverse is also true which gives a necessary and sufficient condition for $h_{c}>0$. If $h_{c}=0$, integrating between $f+h_{c}$ and $f+h$ the equation (23):

$$
\boldsymbol{\nabla}_{\|}(f+h)\left(h-\frac{B i}{\left|\boldsymbol{\nabla}_{\|}(f+h)\right|}\right)=D a_{\|}^{-1} \int_{f}^{f+h} \mathbf{f}_{\|} \mathrm{d} z
$$

Taking the norm and using the threshold condition from (26), we obtain:

$$
|h| \nabla_{\|}(f+h)|-B i|=D a_{\|}^{-1}\left|\int_{f}^{f+h} \mathbf{f}_{\|} \mathrm{d} z\right| \leqslant D a_{\|}^{-1} \int_{f}^{f+h}\left|\mathbf{f}_{\|}\right| \mathrm{d} z \leqslant D a_{\|}^{-1} B i_{p, \|} h
$$

By definition, $h\left|\boldsymbol{\nabla}_{\|}(f+h)\right|-B i \leqslant|h| \nabla_{\|}(f+h)|-B i|$ which gives, with the inequality $(38)$,

$$
h\left(\left|\nabla_{\|}(f+h)\right|-D a_{\|}^{-1} B i_{p, \|}\right) \leqslant B i .
$$

Defined for all $z \in] 0, h]$ and $h, \xi$ in $\mathbb{R}^{+}$the function:

$$
F_{h, \xi}(z)=B i \cosh \left(D a_{\|}^{-1 / 2} z\right)+(z-h)\left(\xi-D a_{\|}^{-1} B i_{p, \|}\right)
$$

Note that the function $z \longmapsto F_{h, \xi}(z)$ is an invertible function on $[0, h]$ when $\left(\xi-D a_{\|}^{-1} B i_{p, \|}\right)>0$. Using the equivalent $h_{c}>0 \Leftrightarrow h\left(\left|\nabla_{\|}(f+h)\right|-D a_{\|}^{-1} B i_{p, \|}\right)>B i$ leads to a general expression for $h_{c}=h_{c}\left(h,\left|\nabla_{\|}(f+h)\right|\right)$ where

$$
h_{c}(h, \xi)=\left\{\begin{array}{cc}
0 & \text { when } h\left(\xi-D a_{\|}^{-1} B i_{p, \|}\right) \leqslant B i \\
F_{h, \xi}^{-1}(0) & \text { when } h\left(\xi-D a_{\|}^{-1} B i_{p, \|}\right)>B i .
\end{array}\right.
$$

In practice, the value of $h_{c}$ for a given $h$ and $\xi$ is efficiently computed by a Newton's algorithm and the machine precision is reached in few iterations. 
Finally, the general expression of $g$ can be written:

$$
g(z)= \begin{cases}\left(\frac{B i_{p, \|}}{\left|\nabla_{\|}(f+h)\right|}-D a_{\|}\right)\left(\frac{\cosh \left(D a_{\|}^{-1 / 2}\left(f+h_{c}-z\right)\right)}{\cosh \left(D a_{\|}^{-1 / 2} h_{c}\right)}-1\right) & \text { when } z \in\left[f, f+h_{c}\right], \\ \left(\frac{B i_{p, \|}}{\left|\nabla_{\|}(f+h)\right|}-D a_{\|}\right)\left(\frac{1-\cosh \left(D a_{\|}^{-1 / 2} h_{c}\right)}{\cosh \left(D a_{\|}^{-1 / 2} h_{c}\right)}\right) & \text { when } \left.z \in] f+h_{c}, f+h\right] .\end{cases}
$$

The last component of the velocity is obtained by integrating the mass conservation $(13 \mathrm{~g})$ in $[f, z]$ :

$$
\int_{f}^{z} \partial_{x} u_{x} \mathrm{~d} z+\int_{f}^{z} \partial_{y} u_{y} \mathrm{~d} z+\int_{f}^{z} \partial_{z} u_{z} \mathrm{~d} z=0
$$

The condition $u_{z}=w_{e}$ in $z=f$ gives:

$$
u_{z}(t, x, y, z)=w_{e}-\int_{f(x, y)}^{z} \operatorname{div}_{\|}\left(\mathbf{u}_{\|}\right) \mathrm{d} z
$$

Thus, velocity $\mathbf{u}$ is an explicit expression depending only upon $h$. Then, the complete stress $\boldsymbol{\tau}$ follows explicitly from (16). It remains to obtain a characterization of $h$ alone. Let us consider (42) at $z=f+h$ : by swapping the derivation $\partial_{x}$ and $\partial_{y}$ with the integral over $[f(x, y), f(x, y)+h(t, x, y)]$, and using the no-slip boundary condition at $z=f$, we obtain:

$$
\int_{f}^{f+h} \partial_{\alpha} u_{\alpha} \mathrm{d} z=\partial_{\alpha}\left(\int_{f}^{f+h} u_{\alpha} \mathrm{d} z\right)-u_{\alpha}(t, x, y, f+h) \partial_{\alpha}(f+h), \forall \alpha \in\{x, y\}
$$

Combining the previous relation with the transport equation (15) at the zeroth order, and replacing in (42) at $z=f+h$, leads to:

$$
\partial_{t} h+\operatorname{div}_{\|}\left(\int_{f}^{f+h} \mathbf{u}_{\|} \mathrm{d} z\right)=w_{e}
$$

By replacing in the previous equation $\mathbf{u}_{\|}=-g \boldsymbol{\nabla}_{\|}(f+h)$ by its expression where $g$ is given in (40), depending only upon $h$, we obtain, after rearrangements, the following conservative equation for $h$ :

$$
\left.\partial_{t} h-\operatorname{div}_{\|}\left\{\tilde{\mu}\left(h,\left|\nabla_{\|}(f+h)\right|\right) \nabla_{\|}(f+h)\right\}=w_{e} \text { in }\right] 0,+\infty[\times \Omega
$$

Here, $\tilde{\mu}$ denotes a diffusion coefficient, defined for all $h, \xi \in \mathbb{R}^{+}$by:

$$
\tilde{\mu}(h, \xi)=\left\{\begin{array}{rr}
\left(D a_{\|}-\frac{B i_{p, \|}}{\xi}\right)\left[\left(h-\frac{B i}{\xi-D a_{\|}^{-1} B i_{p, \|}}\right)-D a_{\|}^{1 / 2} \tanh \left(D a_{\|}^{-1 / 2} h_{c}(h, \xi)\right)\right] \\
0 & \text { when } h_{c}(h, \xi)>0, \\
\text { otherwise. }
\end{array}\right.
$$

This expression contains three parameters: $B i$ from the dimensionless Bingham model and $B i_{p, \|}$ and $D a_{\|}$from the dimensionless Darcy source term added in the momentum 
equation. The no-slip velocity condition at the lateral boundaries leads to an homogeneous Neumann boundary condition:

$$
\left.\frac{\partial(f+h)}{\partial n}=0 \text { on }\right] 0,+\infty[\times \partial \Omega
$$

where $\partial / \partial n=\mathbf{n} \cdot \nabla_{\|}$and $\mathbf{n}$ denotes the outward unit normal on $\partial \Omega$ in the $O x y$ plane. Recall the initial condition:

$$
h(t=0)=h_{\text {init }} \text { in } \Omega
$$

The reduced problem can be written: find $h(t, x, y)$, defined for all $t>0$ and $(x, y) \in \Omega$ and satisfying (43), (45) and (46).

Notice that, for a Newtonian flow $(B i=0)$, expression (44) simplifies as:

$$
\tilde{\mu}(h, \xi)=D a_{\|}\left[h-D a_{\|}^{1 / 2} \tanh \left(D a_{\|}^{-1 / 2} h\right)\right] .
$$

In the present model with the porous zone is consistent with the model without porous zone developed in [5] (i.e. when $D a_{\|}^{-1}=0$ and $B i_{p, \|}=0$ ). Indeed, the expression of $\mu$ is obtained by assuming that $B i_{p, \|}=0$ and by using a truncated expansion in $r=D a_{\|}^{-1 / 2}$. Note $h_{c}=h_{c, 0}+r h_{c, 1}+r^{2} h_{c, 2}+r^{3} h_{c, 3}+r^{4} h_{c, 4}+o\left(r^{4}\right)$. The relation (37) leads to the following limited development of $h_{c}$ :

$$
h_{c}=h_{c, 0}-\frac{B i h_{c, 0}^{2}}{2\left|\boldsymbol{\nabla}_{\|}(f+h)\right|} r^{2}+\frac{B i h_{c, 0}^{3}}{24\left|\boldsymbol{\nabla}_{\|}(f+h)\right|}\left(\frac{13 B i}{\left|\nabla_{\|}(f+h)\right|}-h\right) r^{4}+o\left(r^{4}\right)
$$

where $h_{c, 0}=h-\frac{B i}{\left|\nabla_{\|}(f+h)\right|}$. The expansion at order four is necessary to obtain that of $\tilde{\mu}$ at order two, which is:

$\tilde{\mu}(h, \xi)= \begin{cases}\frac{(2 h \xi+B i)(h \xi-B i)^{2}}{6 \xi^{3}}+r^{2} \frac{B i(h \xi-B i)^{3}(h \xi-13 B i)}{2 \xi^{5}}+o\left(r^{2}\right) & \text { when } h_{c}>0, \\ 0 & \text { otherwise. }\end{cases}$

By taking the limit when $r$ trends to zero, we retrieve the formula (25) as given in [5] for the Bingham case $(n=1)$.

\subsection{The final reduced problem}

Going back to the dimensional variables, the zeroth order equation (43) for the height $h$ can be written:

$$
\left.\partial_{t} h-\left(\frac{\rho g}{\eta}\right) \operatorname{div}_{\|}\left\{\mu\left(h,\left|\nabla_{\|}(f+h)\right|\right) \nabla_{\|}(f+h)\right\}=w_{e} \text { in }\right] 0,+\infty[\times \Omega,
$$

where $\mu$ denotes a diffusion coefficient, defined for all $h, \xi \in \mathbb{R}^{+}$by:

$$
\mu(h, \xi)=\left\{\begin{array}{rr}
\left(\kappa_{\|}-\frac{\tau_{y} \sqrt{\kappa_{\|} \phi}}{\rho g \alpha_{\|} \xi}\right)\left[\left(h-\frac{\tau_{y} \alpha_{\|} \sqrt{\kappa_{\|}}}{\rho g \sqrt{\kappa_{\|}} \xi-\sqrt{\phi} \tau_{y}}\right)-\sqrt{\kappa_{\|}} \tanh \left(\frac{h_{c}(h, \xi)}{\sqrt{\kappa_{\|}}}\right)\right] \\
0 & \text { when } h_{c}(h, \xi)>0, \\
\text { otherwise }
\end{array}\right.
$$


and where

$$
h_{c}(h, \xi)=\left\{\begin{array}{cc}
0 & \text { when } h\left(\rho g \sqrt{\kappa_{\|}} \alpha_{\|} \xi-\sqrt{\phi} \tau_{y}\right) \leqslant \tau_{y} \\
G_{h, \xi}^{-1}(0) & \text { when } h\left(\rho g \sqrt{\kappa_{\|}} \alpha_{\|} \xi-\sqrt{\phi} \tau_{y}\right)>\tau_{y}
\end{array}\right.
$$

where for all $z \in] 0, h]$ and $h, \xi$ in $\mathbb{R}^{+}$the function $G$ satisfies:

$$
G_{h, \xi}(z)=\sqrt{\kappa_{\|}} \alpha_{\|} \tau_{y} \cosh \left(\frac{z}{\sqrt{\kappa_{\|}}}\right)+(z-h)\left(\sqrt{\kappa_{\|}} \alpha_{\|} \rho g \xi-\sqrt{\phi} \tau_{y}\right) .
$$

Equation (47) is completed by the boundary condition:

$$
\left.\frac{\partial(f+h)}{\partial n}=0 \text { on }\right] 0,+\infty[\times \partial \Omega
$$

and the initial condition:

$$
h(t=0)=h_{\text {init }} \text { in } \Omega .
$$

The others unknowns $p, \mathbf{u}, \boldsymbol{\tau}$ and $\mathbf{f}_{p}$ can be explicitly expressed from $h$. The pressure can be directly expressed from $h$ from equation (22):

$$
p(t, x, y, z)=\rho g(f(x, y)+h(t, x, y)-z) .
$$

Likewise, from (40), the horizontal velocity $\mathbf{u}_{\|}=\left(u_{x}, u_{y}\right)$ can be written $\mathbf{u}_{\|}=$ $-g(z) \boldsymbol{\nabla}_{\|}(f+h)$ with:

$$
g(z)= \begin{cases}\frac{\rho g}{\eta}\left(\frac{\tau_{y} \sqrt{\kappa_{\|} \phi}}{\rho g \alpha_{\|} \xi}-\kappa_{\|}\right)\left(\frac{\cosh \left(\frac{f+h_{c}-z}{\sqrt{\kappa_{\|}}}\right)}{\cosh \left(\frac{h_{c}}{\sqrt{\kappa_{\|}}}\right)}-1\right) & \text { when } z \in\left[f, f+h_{c}\right], . \\ \left.\left.\frac{\rho g}{\eta}\left(\frac{\tau_{y} \sqrt{\kappa_{\|} \phi}}{\rho g \alpha_{\|} \xi}-\kappa_{\|}\right)\left(\frac{1-\cosh \left(\frac{h_{c}}{\sqrt{\kappa_{\|}}}\right)}{\cosh \left(\frac{h_{c}}{\sqrt{\kappa_{\|}}}\right)}\right) \quad \text { when } z \in\right] f+h_{c}, f+h\right] . & \end{cases}
$$

From (42) and the previous expression of $\mathbf{u}_{\|}$, the vertical velocity can be computed as:

$$
u_{z}=w_{e}-\operatorname{div}_{\|}\left(\int_{f}^{z} \mathbf{u}_{\|} \mathrm{d} z\right)
$$

From (16) and the previous expressions of $\mathbf{u}_{\|}$and $u_{z}$, the components of the tensor $\boldsymbol{\tau}$ are written: 


$$
\begin{aligned}
\tau_{\alpha z} & =\left[\frac{\tau_{y}}{\left|\partial_{z} \mathbf{u}_{\|}\right|}+\eta\right] \partial_{z} u_{\alpha}, \quad \forall \alpha \in\{x, y\}, \\
\tau_{\alpha \beta} & =\left[\frac{\tau_{y}}{\left|\partial_{z} \mathbf{u}_{\|}\right|}+\eta\right]\left(\partial_{\beta} u_{\alpha}+\partial_{\alpha} u_{\beta}\right), \quad \forall \alpha, \beta \in\{x, y\}, \\
\tau_{z z} & =2\left[\frac{\tau_{y}}{\left|\partial_{z} \mathbf{u}_{\|}\right|}+\eta\right] \partial_{z} u_{z},
\end{aligned}
$$

when $z \in\left[f, f+h_{c}\right]$ and

$$
\left|\boldsymbol{\tau}_{\|}\right| \leqslant \tau_{y}, \quad \text { otherwise. }
$$

Finally, from (17), Darcy's force becomes:

$$
\mathbf{f}_{p, \|}=-\left(\frac{\tau_{y} \sqrt{\kappa_{\|} \phi}}{\kappa_{\|} \alpha_{\|}\left|\mathbf{u}_{\|}\right|}+\frac{\eta}{\kappa_{\|}}\right) \mathbf{u}_{\|},
$$

for the horizontal components and

$$
\mathbf{f}_{p, z}=-\left(\frac{\tau_{y} \sqrt{\kappa_{\|} \phi}}{\kappa_{\perp} \alpha_{\|}\left|\mathbf{u}_{\|}\right|}+\frac{\eta}{\kappa_{\perp}}\right) u_{z}
$$

for the vertical component, when $h_{c}>0$, and

$$
\left|\mathbf{f}_{p, \|}\right| \leqslant \frac{\tau_{y} \sqrt{\kappa_{\|} \phi}}{\kappa_{\|} \alpha_{\|}} \text {when } h_{c}=0 .
$$

\section{$3 \quad$ Numerical method}

\subsection{A new dimensionless formulation}

The nonlinear parabolic problem is first discretized in time by a full implicit second order variable step scheme. At each time-step, the subproblem is linearized by a Newton's algorithm. The resulting subproblems are discretized in space by a finite element method. From a computational point of view, it is convenient to consider a new dimensionless formulation. This second dimensionless procedure differs from the previous one as $\varepsilon$ does no longer appear in the zeroth order problem: the new dimensionless quantities are denoted with an hat. Let $H$ be a characteristic length of the problem and let:

$$
\hat{h}=\frac{h}{H}, \hat{x}=\frac{x}{H}, \hat{y}=\frac{y}{H}, \hat{z}=\frac{z}{H}, \hat{t}=\frac{t}{T}, \hat{f}=\frac{f}{H}, \widehat{w}_{e}=\frac{w_{e}}{U}
$$


where $T=\frac{\eta}{\rho g H}$ represents a characteristic time and $U=\frac{\rho g H^{2}}{\eta}$ a characteristic velocity. After variable substitution, we obtain the following zeroth order dimensionless equation:

$$
\left.\partial_{\hat{t}} \hat{h}-\widehat{\operatorname{div}}_{\|}\left\{\hat{\mu}\left(\hat{h},\left|\widehat{\nabla}_{\|}(\hat{f}+\hat{h})\right|\right) \widehat{\nabla}_{\|}(\hat{f}+\hat{h})\right\}=\widehat{w}_{e} \text { in }\right] 0,+\infty[\times \widehat{\Omega}
$$

where $\hat{\mu}$ is given by (44) by replacing $D a_{\|}, B i$ and $B i_{p, \|}$ by the following new dimensionless numbers:

$$
\widehat{D a}_{\|}=\frac{\kappa_{\|}}{H^{2}}, \widehat{B i}=\frac{\tau_{y}}{\rho g H}, \widehat{B i} i_{p, \|}=\frac{\sqrt{\kappa_{\|} \phi}}{H \alpha_{\|}} \widehat{B i}
$$

As we now only consider this dimensionless problem, and since there is no ambiguity, we omit the hat for all the quantities and also for the dimensionless numbers.

\subsection{A second order implicit time scheme}

Let $\left(t_{m}\right)_{m \geqslant 0}$ the discrete times and $\Delta t_{m}=t_{m+1}-t_{m}, m \geqslant 0$ the corresponding time steps. As the observed solutions decrease exponentially to an arrested state, we choose a geometric progression for the time step $\Delta t_{m+1}=\theta \Delta t_{m}$ where $\theta>1$ and $\Delta t_{0}$ are given.

The time derivative is approximated by the following backward second order variable step finite difference scheme (BDF2), defined for all $\varphi \in C^{0}$ by:

$$
\begin{gathered}
\frac{\partial \varphi}{\partial t}\left(t_{m+1}\right)=\frac{2 \Delta t_{m}+\Delta t_{m-1}}{\Delta t_{m}\left(\Delta t_{m}+\Delta t_{m-1}\right)} \varphi\left(t_{m+1}\right)-\frac{\Delta t_{m}+\Delta t_{m-1}}{\Delta t_{m} \Delta t_{m-1}} \varphi\left(t_{m}\right) \\
+\frac{\Delta t_{m}}{\left(\Delta t_{m}+\Delta t_{m-1}\right) \Delta t_{m-1}} \varphi\left(t_{m-1}\right)+\mathcal{O}\left(\Delta t_{m}^{2}+\Delta t_{m-1}^{2}\right) .
\end{gathered}
$$

The approximate solution sequence $\left(h_{m}\right)_{m \geqslant 0}, h_{m} \approx h\left(t_{m}\right)$, is defined recursively, for all $m \geqslant 1$ by:

$(P)_{m}: h^{m-1}$ and $h^{m}$ being known, find $h^{m+1}$ such that:

$$
\begin{aligned}
\alpha_{m} h^{m+1}-\operatorname{div}_{\|}\left\{\mu\left(h^{m+1},\left|\nabla_{\|}\left(f+h^{m+1}\right)\right|\right) \nabla_{\|}\left(f+h^{m+1}\right)\right\} & =g_{m}+w_{e} \text { in } \Omega \\
\frac{\partial\left(f+h^{m+1}\right)}{\partial n} & =0 \text { on } \partial \Omega
\end{aligned}
$$

where

$$
\begin{aligned}
\alpha_{m} & =\frac{2 \Delta t_{m}+\Delta t_{m-1}}{\Delta t_{m}\left(\Delta t_{m}+\Delta t_{m-1}\right)} \\
g_{m} & =\frac{\Delta t_{m}+\Delta t_{m-1}}{\Delta t_{m} \Delta t_{m-1}} h^{m}-\frac{\Delta t_{m}}{\left(\Delta t_{m}+\Delta t_{m-1}\right) \Delta t_{m-1}} h^{m-1}
\end{aligned}
$$

The sequence is initiated by $h^{-1}=h^{0}=h_{\text {init }}$ for $m=-1$ and 0 , respectively and stopped when $\left\|\left(\frac{\partial h}{\partial t}\right)_{m}\right\|_{L^{2}}<\epsilon$ where $\left(\frac{\partial h}{\partial t}\right)_{m}$ is the approximation by means of the BDF2 scheme of $\frac{\partial h}{\partial t}\left(t_{m}\right)$ and $\epsilon$ is a tolerance that we fixe at $10^{-12}$ for all our simulations. 


\subsection{Newton algorithm}

The initial time-dependent nonlinear parabolic problem is transformed as a sequence of nonlinear subproblems. In [5] an under-relaxed fixed point algorithm is proposed. We present here a new more efficiently Newton algorithm.

Define for all $m \geqslant 0$ and for all $h \in \mathbb{R}^{+}$the function $F_{m}$ :

$$
F_{m}(h)=\alpha_{m} h-\operatorname{div}\left(\mu\left(h,\left|\nabla_{\|}(f+h)\right|\right) \boldsymbol{\nabla}_{\|}(f+h)\right)-\left(g_{m}+w_{e}\right) .
$$

The problem (48) can be equivalently reformulate by using the function $F_{m}$ :

$(P)_{m}: h^{m-1}$ and $h^{m}$ being known, find $h^{m+1}$ such that:

$$
\begin{array}{cc}
F_{m}\left(h_{m+1}\right)=0, & \text { in } \Omega, \\
\frac{\partial\left(f+h_{m+1}\right)}{\partial n}=0, & \text { on } \partial \Omega .
\end{array}
$$

The Newton's algorithm now writes:

Algorithm 1 (Newton).

- $k=0: \varphi^{(0)}:=h_{m}$.

- $k \geqslant 1: \varphi^{(k)}$ known, find $\delta \varphi^{(k)}$ such that:

$$
F_{m}^{\prime}\left(\varphi^{(k)}\right) \delta \varphi^{(k)}=-F_{m}\left(\varphi^{(k)}\right)
$$

- calculate explicitly:

$$
\varphi^{(k+1)}:=\varphi^{(k)}+\delta \varphi^{(k)},
$$

where $F_{m}^{\prime}$ denotes the Fréchet derivative of $F_{m}$.

Note $\psi: \mathbb{R} \longrightarrow \mathbb{R}^{2} ; h \mapsto\left(h,\left|\nabla_{\|}(f+h)\right|\right)$ and $\zeta: \mathbb{R} \longrightarrow \mathbb{R} ; h \mapsto \mu \circ \psi(h)$. With these notations, $\left.F_{m}(h)=\alpha_{m} h-\operatorname{div}(\zeta(h)) \nabla_{\|}(f+h)\right)-\left(g_{m}+w_{e}\right)$. We begin by calculating the Fréchet derivative of $\zeta$. For all $h \in \mathbb{R}^{+}, \delta h \in \mathbb{R}^{+}: \psi^{\prime}(h)(\delta h)=\left(\delta h, \frac{\boldsymbol{\nabla}_{\|}(f+h) \cdot \boldsymbol{\nabla}_{\|}(\delta h)}{\left|\boldsymbol{\nabla}_{\|}(f+h)\right|}\right)$. Likewise, for all $h \in \mathbb{R}^{+}, \xi \in \mathbb{R}^{+}: \delta \mathbf{Z} \in\left(\mathbb{R}^{+}\right)^{2}, \mu^{\prime}(h, \xi)(\boldsymbol{\delta} \mathbf{Z})=\nabla \mu(h, \xi) . \boldsymbol{\delta} \mathbf{Z}$. Finally, according to the rules of the derivatives of the composition of functions:

$$
\begin{aligned}
\zeta^{\prime}(h)(\delta h) & =\mu^{\prime}(h, \xi) \circ \psi^{\prime}(h)(\delta h) \\
& =\nabla \mu(h, \xi) \cdot\left(\delta h, \frac{\nabla_{\|}(f+h) \cdot \nabla_{\|}(\delta h)}{\left|\nabla_{\|}(f+h)\right|}\right),
\end{aligned}
$$


with $\nabla \mu(h, \xi)=\left(\frac{\partial \mu}{\partial h}, \frac{\partial \mu}{\partial \xi}\right)(h, \xi)$ where

$$
\begin{aligned}
\frac{\partial \mu}{\partial h}(h, \xi)= & \left(D a_{\|}-\frac{B i_{p, \|}}{\xi}\right)\left(1-\frac{\partial h_{c}}{\partial h}(h, \xi) \frac{1}{\cosh ^{2}\left(D a_{\|}^{-1 / 2} h_{c}\right)}\right), \\
\frac{\partial \mu}{\partial \xi}(h, \xi)= & \left(D a_{\|}-\frac{B i_{p, \|}}{\xi}\right)\left(\frac{B i}{\left(\xi-D a_{\|}^{-1} B i_{p, \|}\right)^{2}}-\frac{\partial h_{c}}{\partial \xi}(h, \xi) \frac{1}{\cosh ^{2}\left(D a_{\|}^{-1 / 2} h_{c}\right)}\right) \\
& +\frac{B i_{p, \|}}{\xi^{2}}\left(\left(h-\frac{B i}{\xi-D a_{\|}^{-1} B i_{p, \|}}\right)-D a_{\|}^{1 / 2} \tanh \left(D a_{\|}^{-1 / 2} h_{c}\right)\right) .
\end{aligned}
$$

when $h\left(\xi-D a_{\|}^{-1} B i_{p, \|}\right)>B i$ and zero otherwise. The partial derivatives of $h_{c}$ are obtained from the relation on $h_{c}(37)$ :

$$
\begin{aligned}
\frac{\partial h_{c}}{\partial h} & =\frac{\xi-D a_{\|}^{-1} B i_{p, \|}}{D a_{\|}^{-1 / 2} B i \sinh \left(D a_{\|}^{-1 / 2} h_{c}\right)+\xi-D a_{\|}^{-1} B i_{p, \|}} \\
& =\frac{1}{D a_{\|}^{-1 / 2} \tanh \left(D a_{\|}^{-1 / 2} h_{c}\right)\left(h-h_{c}\right)+1}, \\
\frac{\partial h_{c}}{\partial \xi} & =\frac{h-h_{c}}{D a_{\|}^{-1 / 2} B i \sinh \left(D a_{\|}^{-1 / 2} h_{c}\right)+\xi-D a_{\|}^{-1} B i_{p, \|}} \\
& =\frac{h-h_{c}}{\left(\xi-D a_{\|}^{-1} B i_{p, \|}\right)\left(D a_{\|}^{-1 / 2} \tanh \left(D a_{\|}^{-1 / 2} h_{c}\right)\left(h-h_{c}\right)+1\right)} .
\end{aligned}
$$

when $h\left(\xi-D a_{\|}^{-1} B i_{p, \|}\right)>B i$ and 0 otherwise.

Finally, by using these partial derivatives and relation (37) once again:

$$
\begin{aligned}
& \frac{\partial \mu}{\partial h}(h, \xi)=\left(D a_{\|}-\frac{B i_{p, \|}}{\xi}\right) \frac{D a_{\|}^{-1 / 2} \tanh \left(D a_{\|}^{-1 / 2} h_{c}\right)\left(h-h_{c}\right)+\tanh ^{2}\left(D a_{\|}^{-1 / 2} h_{c}\right)}{D a_{\|}^{-1 / 2} \tanh \left(D a_{\|}^{-1 / 2} h_{c}\right)\left(h-h_{c}\right)+1}, \\
& \frac{\partial \mu}{\partial \xi}(h, \xi)= \frac{D a_{\|}\left(h-h_{c}\right)}{\xi} \frac{D a_{\|}^{-1 / 2} \tanh \left(D a_{\|}^{-1 / 2} h_{c}\right)\left(h-h_{c}\right)+\tanh ^{2}\left(D a_{\|}^{-1 / 2} h_{c}\right)}{D a_{\|}^{-1 / 2} \tanh \left(D a_{\|}^{-1 / 2} h_{c}\right)\left(h-h_{c}\right)+1} \\
&+\frac{B i_{p, \|}}{\xi^{2}}\left(\left(h-\frac{B i}{\xi-D a_{\|}^{-1} B i_{p, \|}}\right)-D a_{\|}^{1 / 2} \tanh \left(D a_{\|}^{-1 / 2} h_{c}\right)\right) .
\end{aligned}
$$

when $h\left(\xi-D a_{\|}^{-1} B i_{p, \|}\right)>B i$ and zero otherwise.

We can now write the explicit strong formulation of the linear tangent problem (LT): 
- (LT) Let $\varphi$ know, find $\delta \varphi$ defined on $\Omega$ such that

$$
\begin{aligned}
\alpha_{m} \delta \varphi-\operatorname{div}( & \mu\left(\varphi,\left|\nabla_{\|}(f+\varphi)\right|\right) \boldsymbol{\nabla}_{\|} \delta \varphi+\frac{\partial \mu}{\partial h}\left(\varphi,\left|\nabla_{\|}(f+\varphi)\right|\right) \delta \varphi \boldsymbol{\nabla}_{\|}(f+\varphi) \\
+ & \left.\frac{\partial \mu}{\partial \xi}\left(\varphi,\left|\nabla_{\|}(f+\varphi)\right|\right) \frac{\boldsymbol{\nabla}_{\|}(f+\varphi) \cdot \nabla_{\|} \delta \varphi}{\left|\nabla_{\|}(f+\varphi)\right|} \nabla_{\|}(f+\varphi)\right)=-F_{m}\left(\varphi^{(k)}\right) \\
& \frac{\partial(f+\delta \varphi)}{\partial n}=0 \text { on } \partial \Omega .
\end{aligned}
$$

This linear subproblem is completely standard and is efficiently solved by a piecewise quadratic finite element method, as provided by the Rheolef library [35]. The numerical solution is obtained by solving the linear tangent problem in this weak form (WLT):

- (WLT) find $\delta \varphi \in W^{1, \infty}(\Omega)$ such that:

$$
a_{1}(\varphi ; \delta \varphi, \delta \chi)=l_{1}(\delta \chi), \quad \forall \delta \chi \in W^{1, \infty}(\Omega),
$$

where $a_{1}(. ; .,$.$) and l_{1}($.$) are defined for all \varphi, \delta \varphi, \delta \chi$ in $W^{1, \infty}(\Omega)$ by:

$$
\begin{aligned}
a_{1}(\varphi ; \delta \varphi, \delta \chi)= & \int_{\Omega}\left(\alpha_{m} \delta \varphi \delta \chi+\mu\left(\varphi,\left|\nabla_{\|}(f+\varphi)\right|\right) \nabla_{\|} \delta \varphi \cdot \nabla_{\|} \delta \chi\right. \\
& +\frac{\partial \mu}{\partial h}\left(\varphi,\left|\nabla_{\|}(f+\varphi)\right|\right) \delta \varphi \nabla_{\|}(f+\varphi) \cdot \nabla_{\|} \delta \chi \\
& \left.+\frac{\partial \mu}{\partial \xi}\left(\varphi,\left|\nabla_{\|}(f+\varphi)\right|\right) \frac{1}{\left|\nabla_{\|}(f+\varphi)\right|}\left(\nabla_{\|}(f+\varphi) \cdot \nabla_{\|} \delta \varphi\right)\left(\nabla_{\|}(f+\varphi) \cdot \nabla_{\|} \delta \chi\right)\right) \mathrm{d} x \\
l_{1}(\delta \chi)=- & \int_{\Omega} r \delta \chi \mathrm{d} x
\end{aligned}
$$

Note, for all $\varphi \in \mathbb{R}^{+}$and $\boldsymbol{\xi} \in \mathbb{R}^{2}$ :

$$
\begin{aligned}
\boldsymbol{\beta}(\varphi, \boldsymbol{\xi}) & =\frac{\partial \mu}{\partial h}(\varphi,|\boldsymbol{\xi}|) \boldsymbol{\xi}, \\
k(\varphi, \boldsymbol{\xi}) & =\left(\mu(\varphi,|\boldsymbol{\xi}|) \mathbf{I}_{2}+\frac{\partial \mu}{\partial \xi}(\varphi,|\boldsymbol{\xi}|) \frac{1}{|\boldsymbol{\xi}|} \boldsymbol{\xi} \otimes \boldsymbol{\xi}\right),
\end{aligned}
$$

where $\mathbf{I}_{2}$ is the identity matrix $2 \times 2$. Thus $a_{1}$ can be written with a more compact form:

$a_{1}(\varphi ; \delta \varphi, \delta \chi)=\int_{\Omega} \alpha_{m} \delta \varphi \delta \chi+\left(\delta \varphi \boldsymbol{\beta}\left(\varphi, \nabla_{\|}(f+\varphi)\right)+k\left(\varphi, \nabla_{\|}(f+\varphi)\right) \boldsymbol{\nabla}_{\|} \delta \varphi\right) \cdot \boldsymbol{\nabla}_{\|} \delta \chi \mathrm{d} x$.

\section{Results and discussion}

\subsection{Comparison with Vasilic's experiment [41]}

Vasilic's experiment results are taken from [41]. During the experiment, 12 liters of Carbopol gel are slowly poured into a $20 \times 20 \times 60 \mathrm{~cm}$ transparent container. An array of $d$ 
(a)

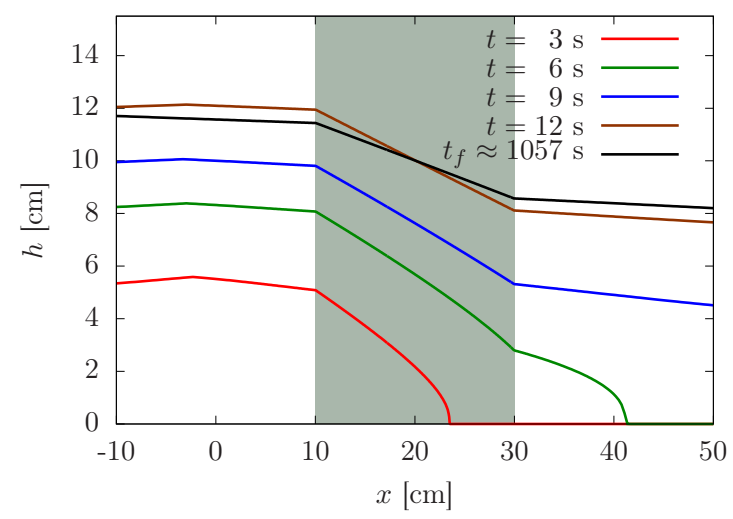

(b)

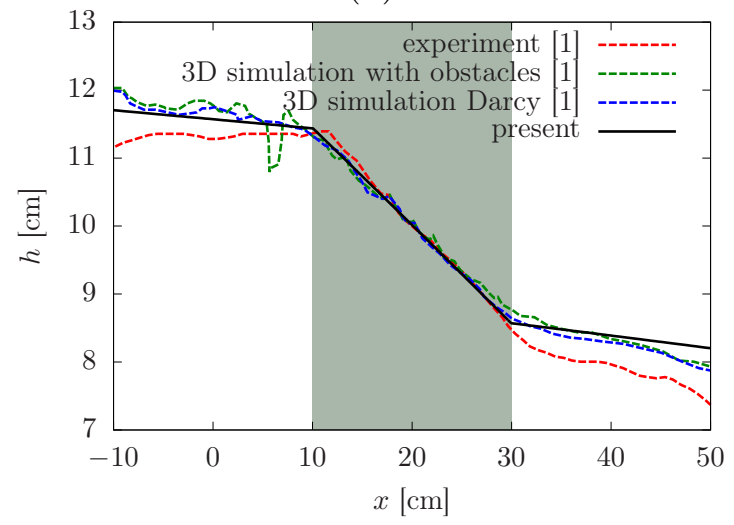

Figure 2: Views of the flow height along the container: (a) Flow heights at different times $t=3 \mathrm{~s}, 6 \mathrm{~s}, 9 \mathrm{~s}, 12 \mathrm{~s}$ and the final time $t_{f} \approx 1057 \mathrm{~s}$ (obtained from BDF2 algorithm and the stopping condition); (b) comparison of the final height between present model and both observations and 3D simulations from [41]. The grey band represents the porous zone.

$=3 \mathrm{~mm}$ cylindrical steel bars is located in the middle zone and spaced at intervals of 19 $\mathrm{mm}$ in the two horizontal directions. The yield stress of this material was measured to be $15 \mathrm{~Pa}$, while its plastic viscosity was measured to be around $1 \mathrm{~Pa} \cdot \mathrm{s}$. The obtained value for the horizontal permeability components is $\kappa_{\|}=6.34 \cdot 10^{-5} \mathrm{~m}^{2}$ and $\alpha_{\|}=1.5$ for the horizontal shift factor. The material flow through the funnel is replaced in our simulations by an equivalent injection. The total volume is injected with a supply rate of $Q$ during a time $t_{e}$ by means of an imposed vertical speed $w_{e}$ through a vent. We take a circular vent with radius $r_{e} \mathrm{~cm}$ centered at the flow origin, and a second order polynomial speed $w_{e}$ versus the radius in the vent which is written:

$$
w_{e}(t, r)= \begin{cases}\frac{2 Q}{\pi r_{e}^{4}}\left(r_{e}^{2}-r^{2}\right)^{+} & \text {if } t \leqslant t_{e} \\ 0 & \text { if } t>t_{e}\end{cases}
$$

The values used in simulations are $Q=1 \mathrm{l} / \mathrm{s}, r_{e}=5 \mathrm{~cm}$ and $t_{e}=12 \mathrm{~s}$.

Fig. 2 (a) shows the shape of the flow at different times. Observe that the porous zone has an effect on the spreading. After $12 \mathrm{~s}$, the flow is no longer supplied, but it is slowly still moving. Fig. 2 (b) compares our results with both laboratory experiments and 3D simulations from [41], using a regularized Bingham model. Results are shown at the final arrested state. Notice that, neglecting lateral wall effects, our problem here still reduces to a $1 \mathrm{D}$ time dependent one. We see a good quantitative agreement of the present approach with both experimental observations and 3D simulations, especially for the slope of the flow front within the porous zone. The discrepancies that can be observed before and after the porous zone, might be a result of wall effects during the experiment. 


\subsection{Flow on a sloping plane for different porous mediums characteristics}

We present here results for a new numerical experiment. Different simulations have been carried out for a flow supplied on an sloping plane and moving through a porous zone. The rheological parameters used are $\eta=1 \mathrm{~Pa} \cdot \mathrm{s}$ and $\tau_{y}=20 \mathrm{~Pa}$. Two angles of inclination have been employed: $\theta=5^{\circ}$ and $\theta=10^{\circ}$. Different porous medium configurations have been investigated. Their characteristics have been defined from different geometrical configurations of periodic arrays of vertical cylinders. By noting $d$ the diameter of the cylinders, $M$ the distance which separates them, and $X=M / d$ the relative distance, the porosity value is $\phi=1-a /(1+X)^{2}$ where $a$ is the fiber arrangement, $a=\pi / 4$ for square packing and $a=\pi /(2 \sqrt{3})$ for hexagonal packing. The permeability can be computed by numerical simulation using Newtonian fluid as proposed in [42] or approximated by a formula such as the law of Tamayol and Bahrami [40] where the horizontal permeability is a function of the porosity and the fiber arrangement:

$$
\kappa_{\|}(\phi)=d^{2} 0.16 a \frac{(1-\sqrt{(1-\phi) / a})^{3}}{(1-\phi) \sqrt{\phi}} .
$$

The simulations have been completed for a square packing $(a=\pi / 4)$ of $d=3 \mathrm{~mm}$ cylinders. The total injected volume is 6 liters by means of an imposed vertical speed $w_{e}$ following the law of (50) with a supply rate of $Q=1 \mathrm{l} / \mathrm{s}$, a vent radius $r_{e}=5 \mathrm{~cm}$ and an injection duration $t_{e}=6 \mathrm{~s}$.

Fig. 3 gives a qualitative comparison of the final state after flow stops for two simulations, with an angle of $\theta=5^{\circ}$ and in (a) without porous zone (denoted by the label $X=\infty$ ) and in (b) with $X=6$. The permeability is calculated by the Tamayol and Bahrami's law. We observe that the presence of a porous zone changes completely the shape of the flow.

(a) $X=\infty$

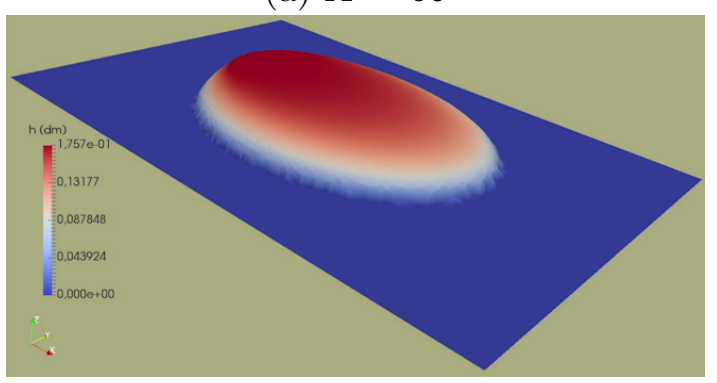

(b) $X=6$

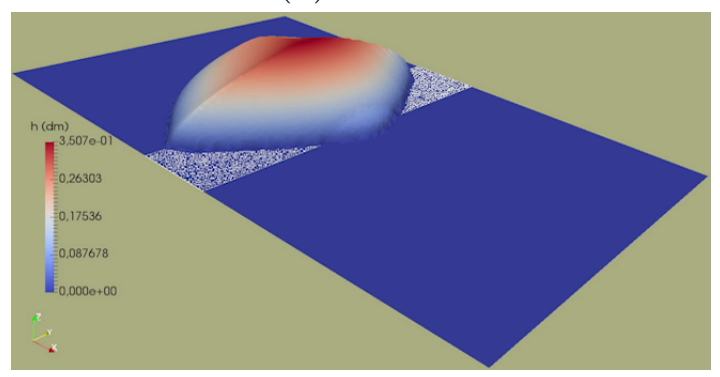

Figure 3: Comparative 3D views for $\theta=5^{\circ}$ and (a) $X=\infty$ (without obstacles) and (b) $X=6$. The white zone on (b) represents the porous zone.

For a more quantitative comparison, the final height along the symmetry line and the final front contours for a range of relative distances $X$ are shown on Fig. 4. We observe on Fig. 4 that the greater the distance between the obstacles, the more the flow is close to 
the case without obstacles $(X=\infty)$. When the distance decreases, we observe from the front views in Fig. 4 (a) and (b), that the flow spreads more laterally and less in the slope direction. Observe on 4 (c) and (d) that a higher thickness of fluid piles up in the porous zone. We can conclude from the results of Fig. 4 that the presence of a porous zone has a limiting effect on the spatial flow propagation by widening the spreading on the lateral sides and by accumulating more volume of fluids per unit surface.

(a) $\theta=5^{\circ}$

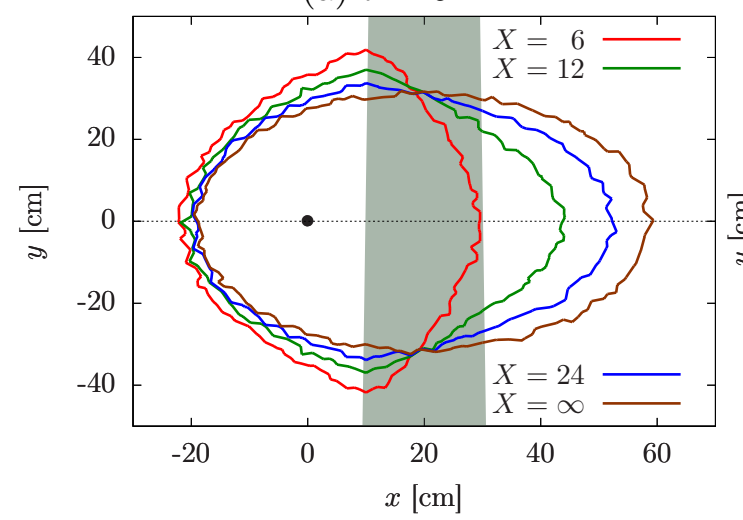

(c) $\theta=5^{\circ}$

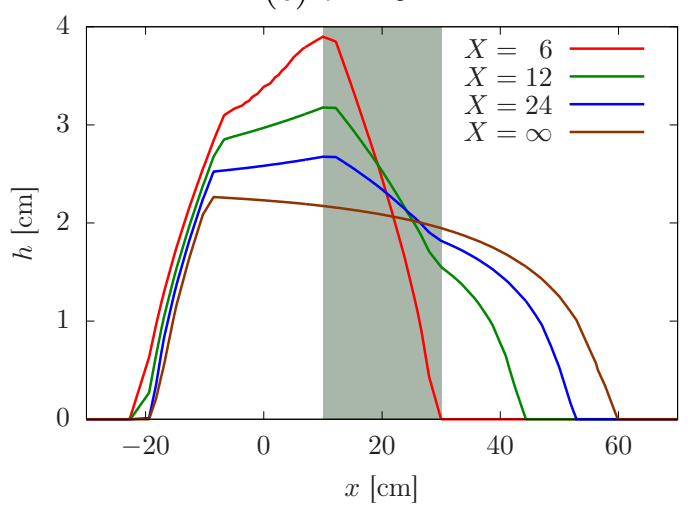

(b) $\theta=10^{\circ}$

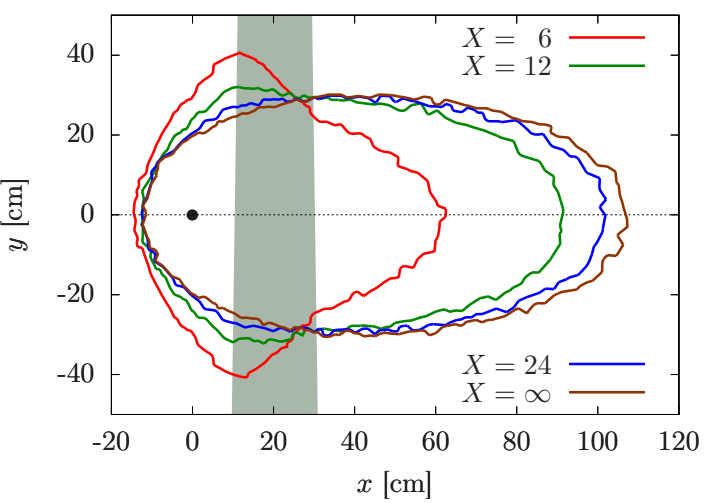

(d) $\theta=10^{\circ}$

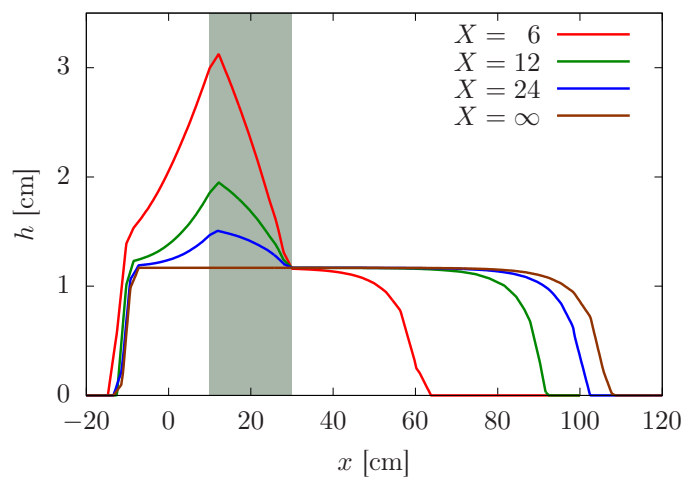

Figure 4: Comparative views for different relatives distances between obstacles $X=$ $6,12,24, \infty$ of the final front contours in (a) for $\theta=5^{\circ}$ and in (b) for $\theta=10^{\circ}$ and the final heights along the symmetry line in (c) for $\theta=5^{\circ}$ and in (d) for $\theta=10^{\circ}$. The grey band represent the porous zone and the dot $\ll \bullet \gg$ the center of the injection.

Fig. 5 shows the maximum flow advance $x_{\max }(t)$ in the slope direction versus the real physical time for several relative distances $X$. We observe that the presence of a porous zone has also a delaying effect by slowing the flow in the slope direction. 
(a) $\theta=5^{\circ}$

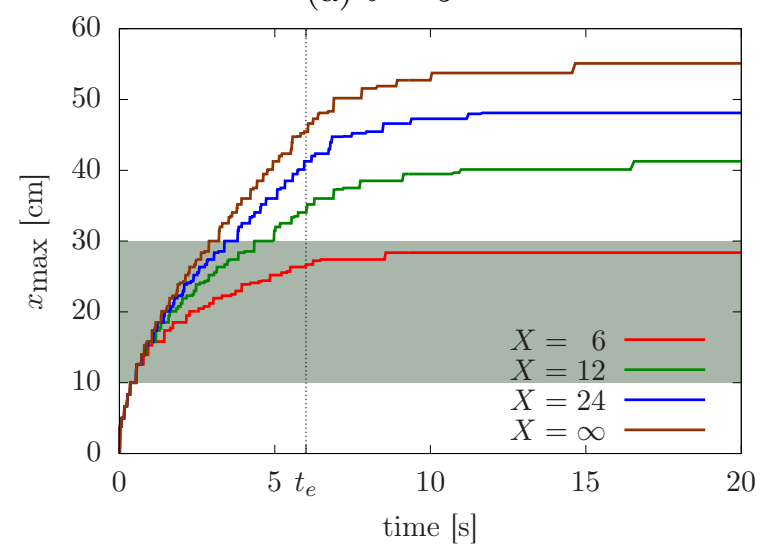

(b) $\theta=10^{\circ}$

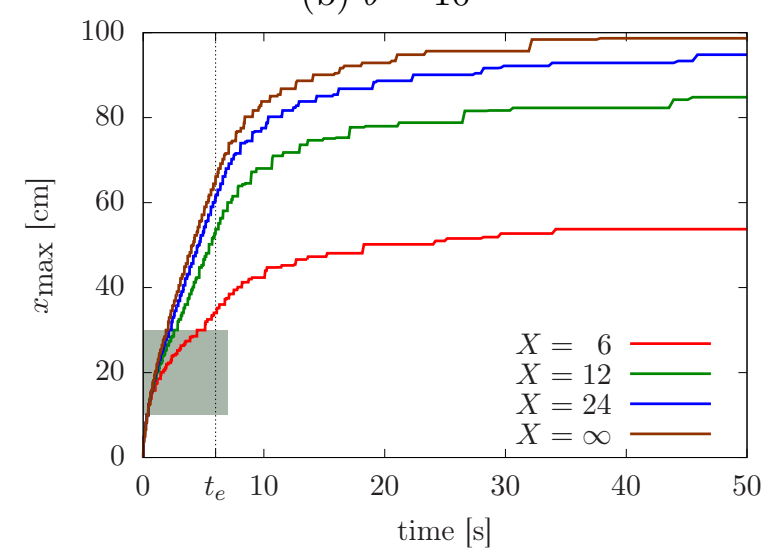

Figure 5: Evolution in time of the maximum flow advance for several relative distances $X=6,12,24, \infty$ with a slope $\theta=5^{\circ}$ in (a) and $\theta=10^{\circ}$ in (b). The grey band shows the passage of the front into the porous zone. The supply duration is $t_{e}=6 \mathrm{~s}$.

\section{Conclusion}

A new reduced model has been developed for laminar shallow viscoplastic fluids flowing on a general topography and crossing an array of vertical obstacles. The model includes two mathematical reductions. The first reduction replaces the obstacles zone by a fibrous media using an extended tensor form of the Brinkman equations for the Bingham rheological behavior. The second reduction in the vertical direction is based on an asymptotic analysis based on the shallow-depth approximation. An efficient numerical resolution using the BDF2 algorithm for time-discretization and a Newton's algorithm for nonlinearities has been developed in Section 3. The sequence of linear subproblems are solved by a finite element method. Finally, two numerical applications are described. A comparison with the measurements and 3D-simulations of [41] shows that the present results are in a good quantitative agreement with analog experiment. The advantage of the present reduced model is that the computational-time is just a few minutes for simulations instead a few hours for 3D-simulations from [41]. We propose also a numerical benchmark concerning the flow of a Bingham fluid over an sloping plane which pass through a fibrous media. The simulations produced for a range of obstacle densities showed the ability of a porous zone to limit the spatial advance of a flow and to delay it. This suggests that, to reproduce this study experimentally and compare with the present results, would inform on the ability of solid,vertical,fixed objects to impede, delay and change the form of a viscous flow. Other further work would be to extend the asymptotic analysis presented here for Bingham material to a Herschel-Bulkley case. For natural applications such as volcanic lava flows through forests, perspectives will be to include complex topographies and thermal effects as developed in [6] and to integrate new thermal effects specific to the trees burning. In this regard, volcanoes are often vegetated, and ingress of lava into such zones will dry, ignite and burn vegetation, and can encase trees in lava to create vertically-oriented solid objects known as "lava trees" [17,27], that would set up a porous array, as modeled here, that could modify and delay the flow. 
Acknowledgments - This work was funded by the Agence National de la Recherche (ANR: http://www.agence-nationale-recherche.fr) through project ANR-LAVA (ANR Program: DS0902 2016; Project: ANR-16 CE39-0009, Link: www. agence-nationale-recherche.fr/?Project=ANR-16-CE39-0009). This is ANR-LAVA contribution no. 4. 


\section{References}

[1] T. Al-Fariss and K. L. Pinder. Flow through porous media of a shear-thinning liquid with yield stress. Canadian J. Chem. Eng., 65(3):391-405, 1987.

[2] D. Andronico, S. Branca, S. Calvari, M. Burton, T. Caltabiano, R. A. Corsaro, P. Del Carlo, G. Garfi, L. Lodato, L. Miraglia, F. Murè, M. Neri, E. Pecora, M. Pompilio, G. Salerno, and L. Spampinato. A multi-disciplinary study of the 2002-03 etna eruption: insights into a complex plumbing system. B. Volcanol., 67(4):314-330, 2005 .

[3] N. J. Balmforth, A. S. Burbidge, R. V. Craster, J. Salzig, and A. Shen. Visco-plastic models of isothermal lava domes. J. Fluid Mech., 403:37-65, 2000.

[4] N. J. Balmforth and R. V. Craster. A consistent thin-layer theory for Bingham plastics. J. Non-Newt. Fluid Mech., 84(1):65-81, 1999.

[5] N. Bernabeu, P. Saramito, and C. Smutek. Numerical modeling of shallow nonNewtonian flows: Part II. Viscoplastic fluids and general tridimensional topographies. Int. J. Numer. Anal. Model., 11(1):213-228, 2014.

[6] N. Bernabeu, P. Saramito, and C. Smutek. Modelling lava flow advance using a shallow-depth approximation for three-dimensional cooling of viscoplastic flows, chapter 27. Geol. Soc., London, 2016.

[7] E. C. Bingham. Fluidity and plasticity. Mc Graw-Hill, New-York, USA, 1922.

[8] J. Bleyer and P. Coussot. Breakage of non-Newtonian character in flow through a porous medium: evidence from numerical simulation. Phys. Rev. E, 89(6):063018, 2014 .

[9] F. Bouchut, A. Mangeney-Castelnau, B. Perthame, and J.-P. Vilotte. A new model of saint venant and savage-hutter type for gravity driven shallow water flows. $C . R$. Math., 336(6):531-536, 2003.

[10] A. Bourgeat and A. Mikelić. Homogenization of a polymer flow through a porous medium. Nonlin. Anal. Theory Meth. Appl., 26(7):1221-1253, 1996.

[11] D. Bresch, E. D. Fernández-Nieto, I. R. Ionescu, and P. Vigneaux. Augmented lagrangian method and compressible visco-plastic flows: applications to shallow dense avalanches. In New directions in mathematical fluid mechanics, pages 57-89. Springer, 2009 .

[12] H. C. Brinkman. A calculation of the viscous force exerted by a flowing fluid on a dense swarm of particles. Appl. Sci. Res., 34:27-34, 1947.

[13] T. Chevalier, C. Chevalier, X. Clain, J. C. Dupla, J. Canou, S. Rodts, and P. Coussot. Darcy's law for yield stress fluid flowing through a porous medium. J. Non-Newt. Fluid Mech., 195:57-66, 2013.

[14] H. P. G. Darcy. Détermination des lois d'écoulement de l'eau à travers le sable, chapter Annexe D, pages 559-603. V. Dalmont, éditeur, Paris, 1856. http://gallica.bnf. fr/ark:/12148/bpt6k624312. 
[15] E. D. Fernández-Nieto, J. M. Gallardo, and P. Vigneaux. Efficient numerical schemes for viscoplastic avalanches. Part 2: the 2D case. J. Comput. Phys., 353:460-490, 2018.

[16] E. D. Fernández-Nieto, P. Noble, and J.-P. Vila. Shallow water equations for power law and bingham fluids. Science China Mathematics, 55(2):277-283, 2012.

[17] R. H. Finch. Lava tree casts and tree molds. Volc Lett, 316:1-3, 1931.

[18] A. Harris, N. Villeneuve, A. Di Muro, V. Ferrazzini, A. Peltier, D. Coppola, M. Favalli, P. Bachèlery, J.-L. Froger, L. Gurioli, S. Moune, I. Vlastelic, B. Galle, and S. Arellano. Effusive crises at piton de la fournaise 2014-2015: a review of a multi-national response model. J. Appl. Volcanol., 6(1):11, 2017.

[19] A. Herault, A. Vicari, A. Ciraudo, and C. Del Negro. Forecasting lava flow hazards during the 2006 etna eruption: using the magflow cellular automata model. Comput. Geo., 35(5):1050-1060, 2009.

[20] D. R. Hewitt, M. Daneshi, N. J. Balmforth, and D. M. Martinez. Obstructed and channelized viscoplastic flow in a Hele-Shaw cell. J. Fluid Mech., 790:173-204, 2016.

[21] G. Hulme. The interpretation of lava flow morphology. Geophys. J. Int., 39(2):361$383,1974$.

[22] I. R. Ionescu. Onset and dynamic shallow flow of a viscoplastic fluid on a plane slope. J. Non-Newt. Fluid Mech., 165(19):1328-1341, 2010.

[23] I. R. Ionescu. Augmented lagrangian for shallow viscoplastic flow with topography. J. Comput. Phys., 242:544-560, 2013.

[24] D. Laigle and P. Coussot. Numerical modeling of mudflows. J. Hydraulic Eng., 123(7):617-623, 1997.

[25] P. W. Lipman, J. P. Lockwood, R. T. Okamura, D. A. Swanson, and K. M. Yamashita. Ground deformation associated with the 1975 magnitude-7.2 earthquake and resulting changes in activity of kilauea volcano, hawaii. Technical report, US Government Printing Office, 1985.

[26] K. F. Liu and C. C. Mei. Approximation equations for the slow spreading of a thin Bingham plastic fluid. Phys. Fluids A, 2(1):30-36, 1990.

[27] J. P. Lockwood and I. S. Williams. Lava trees and tree moulds as indicators of lava flow direction. Geological Magazine, 115(1):69-74, 1978.

[28] S. P. Neuman. Theoretical derivation of darcy's law. Acta Mech., 25(3):153-170, 1977.

[29] H. Pascal. Nonsteady flow through porous media in the presence of a threshold gradient. Acta Mech., 39(3-4):207-224, 1981.

[30] J. R. A. Pearson and P. M. J. Tardy. Models for flow of non-newtonian and complex fluids through porous media. J. Non-Newt. Fluid Mech., 102(2):447-473, 2002.

[31] M. Poreh and C. Elata. An analytical derivation of darcys law. In Israel J. Tech., volume 4, page 214, 1966. 
[32] D. Rickenmann, D. Laigle, B. W. McArdell, and J. Hübl. Comparison of 2D debrisflow simulation models with field events. Comput. Geo., 10(2):241-264, 2006.

[33] B. Robert, A. Harris, L. Gurioli, E. Médard, A. Sehlke, and A. Whittington. Textural and rheological evolution of basalt flowing down a lava channel. B. Volcanol., 76(6):824, 2014.

[34] A. Roustaei, T. Chevalier, L. Talon, and I. A. Frigaard. Non-darcy effects in fracture flows of a yield stress fluid. J. Fluid Mech., 805:222-261, 2016.

[35] P. Saramito. Efficient $C++$ finite element computing with Rheolef. CNRS and LJK, 2015. https://www-ljk.imag.fr/membres/Pierre.Saramito/rheolef.

[36] P. Saramito. Complex fluids: modelling and algorithms. Springer, 2016.

[37] P. Saramito and A. Wachs. Progress in numerical simulation of yield stress fluid flows. J. Rheol., 56(3):211-230, 2017.

[38] S. Shahsavari and G. H. McKinley. Mobility and pore-scale fluid dynamics of ratedependent yield-stress fluids flowing through fibrous porous media. J. Non-Newt. Fluid Mech., 235:76-82, 2016.

[39] K. S. Sorbie, P. J. Clifford, and E. R. W. Jones. The rheology of pseudoplastic fluids in porous media using network modeling. J. Colloid Interface Sci., 130(2):508-534, 1989.

[40] A. Tamayol and M. Bahrami. Transverse permeability of fibrous porous media. Phys. Rev. E, 83(4):046314, 2011.

[41] K. Vasilic. A numerical model for self-compacting concrete flow through reinforced sections: a porous medium analogy. $\mathrm{PhD}$ thesis, faculty of civil engeenering, Dresden, Germany, 2016. https://opus4.kobv.de/opus4-bam/frontdoor/deliver/index/ docId/35783/file/diss144_vt.pdf.

[42] K. Vasilic, B. Meng, H.-C. Kühne, and N. Roussel. Flow of fresh concrete through steel bars: a porous medium analogy. Cement Concrete Res., 41(5):496-503, 2011.

[43] K. Vasilic, W. Schmidt, H.-C. Kühne, F. Haamkens, V. Mechtcherine, and N. Roussel. Flow of fresh concrete through reinforced elements: experimental validation of the porous analogy numerical method. Cement Concrete Res., 88:1-6, 2016. 